\title{
Simulation of Blended Nonlinear Hydrodynamics Forces Using Radial Basis Function in Uniform Moving Frame
}

\author{
M. Saeed Khalid ${ }^{1}$, Salman Nisar ${ }^{1,2 *}$, Sohaib Zia Khan ${ }^{3}$, Muhammad Ali Khan ${ }^{4}$, Armin W. Troesch ${ }^{5}$ \\ ${ }^{1}$ Department of Industrial Manufacturing Engineering and Management, National University of Sciences and Technology, \\ PN Engineering College, PNS Jauhar, Karachi, 74500, Pakistan \\ ${ }^{2}$ Department of Mechanical Engineering, College of Engineering, Taibah University, Madinah, P.O.B 344, Saudi Arabia \\ ${ }^{3}$ Department of Mechanical Engineering, Faculty of Engineering, Islamic University of Madinah, Madinah, P.O.B 170, Saudi Arabia \\ ${ }^{4}$ School of Aerospace, Transport and Manufacturing, Cranfield University, Cranfield, UK \\ ${ }^{5}$ Department of Naval Architecture and Marine Engineering, University of Michigan, Ann Arbor, Michigan, USA \\ *Corresponding author e-mail: snahmed@taibahu.edu.sa
}

\begin{abstract}
This study focuses on the development of a blended technique in moving frame which encompasses nonlinearities and real time simulation of the vital early design parameters using combined exact nonlinear and quasi-nonlinear forcing terms. Generally, a full three-dimensional problem needs to be solved for the precise forward speed correction. However, in this paper the forward speed end corrections are calculated by converting the two dimensional velocity potential into a three dimensional mathematical function using radial basis function then partial differentiation is performed with respect to the longitudinal direction. The difference between the forward speed correction used for time simulation in the blended method and the strip-theory in the frequency domain has been explained. The use of radial basis functions for the estimation of quasi-nonlinear combined radiation and diffraction pressures in moving frame and their conversion between two and three dimensions has been demonstrated and validated experimentally.
\end{abstract}

Keywords: Blended technique; Radial basis function; Strip-theory; Radiation and diffraction pressures; Moving frame 


\section{Nomenclature}

\begin{tabular}{|c|c|}
\hline$U$ & Mean forward speed/velocity \\
\hline$X$ & Ship absolute motions vector \\
\hline$F_{n}$ & Froude number \\
\hline$\widetilde{F_{R}}(t)$ & Complex radiation force time series \\
\hline$\widetilde{F_{D}}(t)$ & Complex wave load force time series \\
\hline$x, y, z$ & Inertial coordinate system \\
\hline$\eta_{1}$ & Surge \\
\hline$\eta_{2}$ & Sway \\
\hline$\eta_{3}$ & Heave \\
\hline$\eta_{4}$ & Roll \\
\hline$\eta_{5}$ & Pitch \\
\hline$\eta_{6}$ & Yaw \\
\hline$x_{0}$ & $\mathrm{X}$-axis in earth fixed coordinate \\
\hline$t_{0}$ & Time in earth fixed coordinate \\
\hline$n$ & Normal vector \\
\hline$t$ & Surface tangent \\
\hline$\varphi R$ & Radiation velocity potential \\
\hline$\varphi D$ & Diffracted velocity potential \\
\hline$\varphi I$ & Incident wave velocity potential \\
\hline$F$ & Force \\
\hline$S$ & Surface/Segment \\
\hline$\Phi$ & Total Velocity potential \\
\hline$\nabla$ & Laplace operator \\
\hline$D$ & Total / cumulative derivative \\
\hline$\varphi$ & Perturbation velocity potential \\
\hline$\varphi_{s}$ & Steady perturbation velocity potential \\
\hline$\varphi_{R}$ & Unsteady perturbation velocity potential \\
\hline$W$ & Velocity flow vector \\
\hline$\Omega$ & Rotational velocity vector \\
\hline$V$ & Ship unsteady oscillatory velocity vector \\
\hline$u, v, w$ & Subscripts used for velocity in $\mathrm{x}, \mathrm{y}$ and $\mathrm{z}$ directions \\
\hline$\Psi(r)$ & Single basis function \\
\hline$\xi$ & Instantaneous wave profile \\
\hline$p$ & Dynamic pressure \\
\hline$\omega_{e}$ & Frequency of encounter \\
\hline$\kappa$ & Wave number \\
\hline$\beta$ & Heading angle \\
\hline$z$ & Complex points on the body surface \\
\hline$z_{o}$ & Complex points in the fluid water domain \\
\hline$L$ & Linear \\
\hline$N$ & Quasi-Nonlinear \\
\hline$E$ & Experimental \\
\hline$r$ & Complex data (gain) \\
\hline$i$ & Complex data (imaginary) \\
\hline
\end{tabular}




\section{Introduction}

Rapid and repetitive assessment of seakeeping parameters is needed during an early design phase of sea-going vessel. Along with the accuracy of modelling, time to complete the assessment cycle also becomes a dominant factor. Therefore, a fast and robust seakeeping code that must be capable of performing rigorous hydrodynamics calculations is always desirable.

Over the past several decades, numerous investigations have been conducted on hydrodynamic analysis of hull behaviour. They are classified into three major categories: analytical, experimental and numerical techniques. The numerical techniques are further divided into methods for boundary value problems and domain-dependent problems. Each technique is applicable only for a limited range of cases [1]. Plethora of publications exists on the subject of BEMs applied for single hull hydrodynamics. Doctor et al. [2] and Wang and Day [3] utilized a distribution of pressure elements over planning surfaces while Lai and Troesch [4] and Benedict et al. [5] applied vortex lattice methods. Several CFD-type analyses of specific high-speed multihulls can be also found in the literature (e.g., Zhou, 2003 [6]; Kandasamy et al., 2011 [7]; Yousefi et al., 2014[8]). Jiang et al. [9] employed CFD code based on finite volume method to simulate and analyse the forward motion of the hull body with two degrees of freedom (heave and pitch). The main objective of the study was to analyse the flow mechanism and characteristics of the tunnel at different forward speeds. Predicted results were validated with experimental data and showed good agreement. Ghassabzadeh and Ghassemi [10] used commercial software FLUENT to simulate a multi-hull tunnel vessel in calm water. The degrees of freedom in the simulation were pitch and heave motions and the hull position changed with a moving mesh. The simulated results of drag and trim were in good agreement with experimental data.

The evaluation of structural responses is key element in the design of ships and off shore structures. The total pressure to examine hull behaviour comprises of incident wave Froude-Kriloff pressure, hydrostatic pressure and radiation and diffraction pressures. Hirdaris et al [11] presented a detailed review on the recent advances in the assessment of loads for ships and off shore structures for further understanding, validation and implementation by the academic and industrial communities.

Many researchers have worked to solve the instantaneous boundary value problem using desingularized Euler-Lagrange methods. Finn [12] solved the boundary value problem which satisfies body boundary condition in the near field. The free surface condition is only satisfied within the reasonably wide range of free space of water surface, in the vicinity of the piercing body. The matching of far field at infinity and near field solutions has not been considered by assuming that the impact of the far field condition on the near field solutions would not be significant.

Kent [13] used a pseudo-spectral method, where asymptotic technique is employed to seek the 
solution that satisfies the far field boundary conditions. This approach is close to the one used by Faltinsen [14] for diffraction velocity potential for a piercing body in head seas where far field and near field conditions are matched asymptotically.

Salvesen et al. [15] used conventional strip theory for seakeeping analysis of normal displacement ships. However, its validity can be questioned when it is used for ships with higher maximum operating speeds, such as for semi-displacement vessels [16].

Savander et al. [17] applied the boundary value problem to a planing plate and obtained relationships between potential perturbation and vortex distribution. They calculated the hydrodynamic pressure, lift and drag forces for the planning plate at different speeds. Ghassemi et al. [18]-[20] have developed a computer code, based on BEM in conjunction with boundary layer, for hydrodynamic analysis of planing and non-planing hulls. This code was also utilized to study the wave pattern and pressure coefficients. However, the code was unable to take into account a two-phase flow model and cannot be applied for complex geometries and high Froude number cases.

Kihara et al. [21] presented computations of hydrodynamic forces when the ship oscillates in head seas, although their focus is on the added resistance in waves and they simplified the description of sprays. They simulated non-viscous flow separation from the knuckle of a ship section using $2 \mathrm{D}+\mathrm{t}$ method together with a nonlinear BEM solver.

Giorgi and Ringwood [22] discussed the importance and relevance of nonlinear Froude Kriloff force representation for axisymmetric wave energy converters, for vessels moving in surge, heave, and pitch. It is found that Froude-Kriloff integrals can be solved numerically by assuming small pitch angles which simplify the problem and achieve a considerably faster algebraic solution.

Yuan et al. [23] investigated the hydrodynamic interactions between two ships arranged side by side with forward speed using boundary element program based on 3-D Rankine source method. The radiation condition is satisfied by using a modified Sommerfeld radiation condition which takes into account the Doppler shift of the scattered waves. This new radiation condition is applicable to a wide range of forward speeds. Yuan et al. [24] also investigated the hydrodynamic interaction between two vessels with forward speed arranged side by side in shallow water. The motion responses of both ships were calculated and compared to these obtained from commercial software and experimental results.

Rajendran et al. [25] investigated the effects of surge hydrodynamic forces and surge mode of motion on the vertical responses of a container ship and a chemical tanker in waves. It was found that the longitudinal forces have insignificant effect on the vertical moment about the lateral axis at the mean water level and on the flexural normal stress at the deck. Rajendran et al. [26] also developed a code based on strip theory to calculate vertical ship responses of a containership in extreme sea conditions. The numerical method calculates the radiation forces based on Cummins formulation. Radiation, diffraction, Froude-Krylov and hydro static forces for instantaneous wetted surface area of the hull were calculated. It is observed that the body nonlinear 
radiation/diffraction forces significantly improve prediction of the ship responses in extreme waves.

Khalid et al. [27] investigated the role of blended method to solve two-dimensional boundary value problem for multi-bodies radiation and diffraction velocity potentials. It was found that blended technique is an efficient and accurate alternate method to provide time simulations of ship motion and other essential parameters for design optimization.

In this paper, blended technique using radial basis function has been developed and employed to calculate hydrodynamic forces in moving frame. This technique encompasses nonlinearities and real time simulation of the vital early design parameters using combined exact nonlinear and quasinonlinear forcing terms. The difference between the forward speed correction used for time simulation in the blended method and the strip-theory in the frequency domain has been explained. The use of radial basis functions for the estimation of quasi-nonlinear combined radiation and diffraction pressures in moving frame and their conversion between two and three dimensions has been demonstrated and validated experimentally.

\section{Radial Basis Function and Convergence Studies}

\subsection{Radial Basis Function}

The functions expressed in the distance variable are usually termed as radial basis functions (RBFs) in the literature. The idea of radial-basis function derives from the theory of functions. A radial-basis function approach is formed by taking a linear combination of a single basis function $\Psi(\mathrm{r})$, which is radially symmetric about its center. The only geometric property used in the radial basis function method is the pairwise distances between points. Wright and Fornberg [28] have shown that the interpolation can be accurate for approximating derivatives. By imposing some mild restrictions on $\Psi(\mathrm{r})$, the radial-basis function interpolation converges to polynomials. The radial basis function is a weighted sum of the radially symmetric basic functions located at the centers/nodes and a low degree polynomial. The radial basis function evaluates the nodal value pressure by keeping track of the new relative position of all the other nodes in the domain of interest. It also saves an enormous amount of time by not solving the boundary value problem at each full and intermediate time step. Wide range of radial basis functions can be found in the literature with different kinds of constraints and utilities such as bi-harmonic, tri-harmonic, quadratic, multi-quadratic, inverse multi-quadratic, wend-land and generalized Duchon spline radially symmetric functions [28]-[31]. Khalid [32] in his thesis evaluated dynamic and static pressures acting on a floating body while the body is experiencing large motions in six degrees of freedom and extreme random seas. A blended scheme is introduced to calculate total hydrodynamic pressure acting on an arbitrary body. The method employs quasi-nonlinear radiation and diffraction models while hydrostatic and Froude-Kirloff pressures are fully nonlinear. The quasi-nonlinear radiation and diffraction pressures are estimated using radial-basis function without solving the boundary-value problems. 


\subsection{Convergence Studies}

In this study, radial-basis function is used for the estimation of body exact instantaneous radiation and diffraction pressures. There are two distinct applications, the first is to capture the changing sectional geometries and the second is to evaluate partial derivative $\frac{\partial}{\partial x}$ of the radiation and diffraction velocity-potentials needed for the evaluation of forward-speed corrections.

Typical convergence investigations are illustrated using real part of a three dimensional incident wave velocity-potential $f(x, y, x)=e^{z} \sin (-x-y)$, where the wave amplitude and wave number are assumed to be unity. The initial radial-basis coefficients $\lambda_{i}$ are evaluated for the mean position of a half-submerged cylinder of radius $R=1$. The cylinder is then further submerged by a factor of $0.4 R$. The previously computed $\lambda$ 's corresponding to mean position are used to estimate the function $f_{r}(x, y, z)$ for the changed body-section; the variation of body geometry is shown in Figure 1.

The extent of change in the body geometry relative to its mean position is also investigated. For the same function and cylindrical body, various emersions and immersions are shown in Figure 2 using 25 nodal points on a section. The estimates compare reasonably well for extreme geometry changes. The estimate for extreme geometry reduction due to emersion of $0.7 R$ of the cylinder is $10 \%$ of the exact value; for an immersion of $-0.7 R$, the error is $30 \%$ of exact value. In general for moderate changes of geometry the error in estimates remains below $3 \%$ of exact values.

Convergence studies for the second application of radial-basis function to evaluate $\frac{\partial}{\partial x}$, is demonstrated using the partial derivative $\frac{\partial f}{\partial x}$ of the previously used function $f(x, y, z)$ for a cylindrical ship-shape. The ship's length equals the length of the wave, i.e $L=\lambda=2 \pi$ and the radius of the each section is $R=\frac{\lambda}{10}$. The exact $\frac{\partial f(x, y, z)}{\partial x}$ is known at different nodes on various sections along the ship's length.

The radial-basis coefficients $\lambda_{i}$ are evaluated for the function $f(x, y, z)$. Then the estimated function $f_{r}(x, y, z)$ is constructed using these coefficients. The partial derivative $d f r=\frac{\partial f_{r}(x, y, z)}{\partial x}$, is performed on the mathematical or algebraic form of the function $f_{r}(x, y, z)$. The estimates are increasingly good for an increase in number of stations and the convergence is fast as shown in Figure 3. The relative error is reasonably small for moderate numbers of stations along the ship's length. 


\section{Mathematical Formulation}

\subsection{Fully Nonlinear Steady Perturbation Problem}

To account for the forward speed corrections, a steady perturbation velocity potential $\varphi_{s}$ is found through solving a steady flow problem in moving frame. The velocity vector $W$ of steady flow relative to the moving frame is defined in Equation 1. Since the dynamic pressure given by Bernoulli's Equation holds for all time, setting its substantial derivative equal to zero gives an alternative fully nonlinear free surface boundary condition (2). Substituting $U\left(\varphi_{s}-x\right)$ for velocity potential $\Phi$ in Eq. (2), one may write the free surface, $z_{o}=\xi(x, y, t)$ boundary and body boundary conditions at mean body position in terms of steady velocity vector $W$ as in Eqs. ( $3 \& 4$ ), where $n$ is unit normal vector. In this text $n$ is vector unless written subscripted as $n_{j}$.

$W=U \nabla\left(\varphi_{s}(x, y, z)-x\right)$

$\frac{\partial^{2} \Phi}{\partial t^{2}}+\nabla \Phi \cdot \nabla \Phi_{t}+\frac{1}{2} \nabla \Phi \cdot \nabla(\nabla \Phi \cdot \nabla \Phi)+g \Phi_{z_{o}}=0 \quad$ on $\quad z_{o}=\xi$

$\frac{1}{2} W \cdot \nabla\left(W^{2}\right)+g \frac{\partial \varphi_{s}}{\partial z_{o}}=0 \quad$ on $\quad z_{o}=\xi$

$W \cdot n=0 \quad$ on $S_{o}$

To include all the nonlinearities, one should solve for the combined steady and unsteady $\varphi_{S R}$ $=\mathrm{U} \varphi_{s}+\varphi_{R}$ velocity potential subject to combined free surface and instantaneous body boundary conditions ( $6 \& 7$ ) in terms of the ship absolute velocity and steady flow vector $W$. However, the assumption of small motions leads to decomposition of unsteady velocity potential $\varphi_{R}$ into its components for each of six degrees of rigid body motions. Under such conditions, one may solve the steady flow problem separately and the unsteady velocity potential separately with the free surface boundary condition (5) and the body boundary condition (7). This is accomplished by replacing velocity flow vector $W$ with $\widehat{U}$ i and neglecting the steady perturbation velocity potential $\varphi_{\mathrm{s}}$

$\left[\left(\frac{\partial}{\partial t}-U \frac{\partial}{\partial x}\right)^{2}+g \frac{\partial}{\partial_{z}}\right] \varphi R=0$

$\frac{\partial^{2} \varphi R}{\partial t^{2}}+2 W \cdot \nabla \frac{\partial \varphi R}{\partial t}+\frac{1}{2}(W+\nabla \varphi R) \cdot \nabla W^{2}$

$+W \cdot \nabla(W \cdot \nabla \varphi R)+g \frac{\partial(\varphi R+\varphi s)}{\partial_{z}}=0 \quad$ on $\quad z_{o}=\xi$

$\frac{\partial \varphi R}{\partial n}=\nabla \varphi R \cdot n=(V-W) \cdot n \quad$ on $\quad S(t)$ 
Newman [33] mentioned two first order contributions of $W \cdot n$ in the instantaneous body boundary condition (7) as (a) rotation of the ship fixed coordinate system and (b) gradient of the steady flow. Therefore, one may express the last term $W \cdot n$ used in Eq. (7) as vectorial representation of coordinate rotation and gradient of the steady flow. Using Eq. (8), where $\Omega$ is the rotational velocity vector representing roll, pitch and yaw velocities and $X$ represents the ship absolute motions vector. After substitution of Eq. (8) into Eq. (7), the body boundary condition (7) can be applied to steady state body position and to the instantaneous body position. Eq. (8) is valid on both steady state and instantaneous body positions. By invoking Eq. (4) and using vector equalities $\Omega \times W=(W \cdot \nabla) X$, the alternative of the Eq. (8) may be written as Eq. (9)

$$
\begin{aligned}
& W \cdot n \cong(W-\Omega \times W+(X \cdot \nabla) W) \cdot n \\
& W \cdot n \cong(-(W \cdot \nabla) X+(X \cdot \nabla) W) \cdot n
\end{aligned}
$$

Bernoulli's equation for dynamic pressure in the coordinate system moving with velocity vector $W$ will be written as in Eq. (10). The radiation velocity potential $\varphi R$ in Eq. (10) should be obtained by solving a boundary value problem subject to fully nonlinear boundary conditions ( 6 \& 7) after substitution of Eq. (9) into the Eq. (7)

$p=-\rho\left(\frac{\partial \varphi R}{\partial t}+W \cdot \nabla \varphi R+\frac{1}{2} \nabla \varphi R \cdot \nabla \varphi R+g z_{o}\right)+p_{a}$

\subsection{Linear Approximation and Decomposition}

To avoid the complexities of the three-dimensional problem, one may tend to neglect the steady flow field due to the ship presence. Assuming $\varphi_{s}=0$ the velocity flow vector will be $\mathrm{W}=-\widehat{U}$, the body boundary condition (7) will reduce to Eq. (11). It is observed here that with the assumption of $\varphi_{s}=0$, one of the two first order contributions of $W \cdot n$ is lost completely. The only first order contribution retained in the body boundary condition is due to the rotation of the coordinate system. The flow velocity vector is constant $\mathrm{W}=-\widehat{U} \mathrm{i}$; its gradient will vanish.

$\frac{\partial \varphi R}{\partial n}=\nabla \varphi R \cdot n=\left(V-U \frac{\partial X}{\partial x}\right) \cdot n \quad$ on $S(t)$

The assumption of small unsteady motions leads to decomposition of $\varphi R$ into separate six degrees of freedom and the body boundary conditions for six cases would be as follows: 
$\frac{\partial \varphi R}{\partial n}=\nabla \varphi R \cdot n=\dot{\eta}_{1} n_{1}$ on $S_{o}$

$\frac{\partial \varphi R}{\partial n}=\nabla \varphi R \cdot n=\dot{\eta}_{2} n_{2}$ on $S_{o}$

$\frac{\partial \varphi R}{\partial n}=\nabla \varphi R \cdot n=\dot{\eta}_{3} n_{3}$ on $S_{o}$

$\frac{\partial \varphi R}{\partial n}=\nabla \varphi R \cdot n=\dot{\eta}_{4} n_{4}$ on $S_{o}$

$\frac{\partial \varphi R}{\partial n}=\nabla \varphi R \cdot n=\dot{\eta}_{5} n_{5}+U \eta_{3} n_{3}$ on $S_{o}$

$\frac{\partial \varphi R}{\partial n}=\nabla \varphi R \cdot n=\dot{\eta}_{5} n_{5}-U \eta_{2} n_{2}$ on $S_{o}$

The term proportional to $U$ may be interpreted as the product of the ship forward speed and angle of attack due to pitch and yaw motions. With the additional restriction that unsteady motions are sinusoidal oscillations with $\omega_{e}$ as the frequency of encounter, the velocities and displacements are related as $\mathrm{V}=i \omega_{e} X$, the Eqs $(16,17)$ and Bernoulli's Eq. (10) may be further linearized as follows:

$$
\begin{aligned}
& \frac{\partial \varphi R}{\partial n}=\nabla \varphi R \cdot n=\dot{\eta}_{5} n_{5}+\frac{U}{i \omega_{e}} \dot{\eta}_{3} n_{3} \text { on } S_{o} \\
& \frac{\partial \varphi R}{\partial n}=\nabla \varphi R \cdot n=\dot{\eta}_{5} n_{5}-\frac{U}{i \omega_{e}} \dot{\eta}_{2} n_{2} \text { on } S_{o} \\
& p=-\rho\left(\frac{\partial \varphi R}{\partial t}-U \frac{\partial \varphi R}{\partial x}+g z_{o}\right)+p_{a} \\
& \frac{\partial \varphi R_{j}}{\partial n}=\nabla \varphi R_{j} \cdot n=i \omega_{e} n_{j} \\
& \left(\frac{\partial^{2}}{\partial t_{o}^{2}}+g \frac{\partial}{\partial z_{o}}\right) \varphi R_{j}=0 \quad \text { at } Z=\zeta
\end{aligned}
$$

The last terms in each of the Eq. ( $18 \& 19)$ represent the body boundary conditions ( $13 \& 14)$ with a constant multiplier $\pm \frac{U}{i \omega_{e}}$ respectively. One may intend to solve the six boundary value problems, without explicit involvement of forward speed, using a simple two-dimensional Green's function given by [34] and subjected to simple free surface and body boundary conditions (21 \& 22). However doing so will result in an inherent anomaly in free surface pressure. The free surface pressure would be slightly different than absolute zero value because of not satisfying the consistent linearized free surface boundary condition (5) involving the forward speed $U$ after neglecting the steady perturbation velocity $\varphi_{s}$ in the boundary value problem. After solving the boundary value problem for $\varphi_{R}^{o}$ using body boundary conditions (12 through 17) with $U=0$ and consistent linearized free surface boundary condition (5), the pitch and yaw radiation potentials then may be corrected for the constant multiplier as shown in Eqs. ( 23 \& 24). There is additional 
forward speed correction present in linearized dynamic pressure Eq. (20) due to moving frame of reference.

$$
\begin{aligned}
& \varphi R_{5} \cong \varphi_{R_{5}}^{o}+\frac{U}{i \omega_{e}} \varphi_{R_{3}}^{o} \\
& \varphi R_{6} \cong \varphi_{R_{6}}^{o}-\frac{U}{i \omega_{e}} \varphi_{R_{2}}^{o}
\end{aligned}
$$

\section{Blended Method for Radiation Forces in Moving Frame}

In blended method, fully nonlinear Euler equations of motion are solved with nonlinear hydrodynamic forces acting on multi-hulled vessels. Lid is employed over the body segment to suppress the eigenvalue mode, thus eliminating singularities in source strength being used on multi-hull bodies presenting geometrical discontinuities [27]. In the nonlinear time simulation, added-mass and damping coefficients are not used. Instead, total nodal radiation pressure is estimated using an appropriate radial-basis function for exact instantaneous body position. Six boundary value problems are solved separately without involving forward speed. Then after correcting the $\varphi R_{j}$ for pitch and yaw modes by invoking the Eqs. ( $\left.23 \& 24\right)$, the total radiation velocity potential in rotating frame may be expressed as shown in Eq. (25). The substitution of this $\varphi R$ in Eq. (20) gives the dynamic pressure in moving-coordinates.

$$
\varphi R=\sum_{j=1}^{6} \varphi R_{j} \eta_{j}
$$

The diffracted velocity potential $\varphi D$ is also solved for simple free surface and body boundary conditions $(21 \& 22)$. For the diffraction boundary value problem, the body is held in steady state position against the incident wave, the forward speed correction $-U \frac{\partial X}{\partial x}$ in the body boundary condition (11) for a rotating frame will vanish, as there is no rotation of the coordinate system. However the forward speed correction $U \frac{\partial}{\partial x}$ of linearized dynamic pressure Eq. (20) due to steady moving-coordinate is absolutely applicable for the diffracted velocity potential as well. Similarly, due to the moving frame of reference for the incident velocity potential, the only forward speed correction needed is $U \frac{\partial}{\partial x}$ in the linearized dynamic pressure Eq. (20). In the blended method, an appropriate radial-basis function is used to convert two-dimensional radiation and diffraction velocity potentials into their three-dimensional equivalents and the partial derivative $U \frac{\partial}{\partial x}$ in Eq. (20) is performed explicitly for the combined radiation and diffracted velocity potentials. 


\subsection{Validations}

For the purpose of validation a comparison is presented between linear strip-theory of [15] and the blended method adopted in this research. In strip theory [15], the added-mass and damping coefficients may be written as in Eq. (26), where $\varphi R_{j}$ has been corrected for rotation of coordinate system as in Eqs. (23 \& 24).

$\omega_{e}^{2} A_{i j}-i \omega_{e} B_{i j}=-\rho \iint\left(i \omega_{e} \varphi R_{j}-U \frac{\partial \varphi R_{j}}{\partial x}\right) n_{i} d S$

For ease of understanding, one may split the $\varphi R_{j}$ into two parts as $R_{j}=\varphi_{R_{j}}^{o}+\varphi_{j}^{u}$. Where $\varphi_{R_{j}}^{o}$ is the solution of the boundary value problem obtained from six body boundary conditions (12 through 17) with $U=0$ and free surface boundary condition (5) and $\varphi_{j}^{u}$ is forward speed correction occurring in the body boundary condition due to rotation of the coordinate system. Considering Eqs. $(23 \& 24)$, the velocity potential $\varphi_{j}^{u}=0$ for $\mathrm{j}=1,2,3,4$ and is nonzero for pitch $\varphi_{5}^{u}=\frac{\varphi_{R_{3}}^{o}}{i \omega_{e}}$

and yaw $\varphi_{6}^{u}=\frac{\varphi_{R_{2}}^{o}}{i \omega_{e}}$ modes.

The forward speed correction for the radiation velocity potential arises because of axes rotation, this correction is applied to the mean position of the body in the strip theory [15] while in the blended method the same correction is estimated for the instantaneous position of the body by using an appropriate radial-basis scheme with similar inconsistencies with regard to free surface boundary condition. The unsteady velocity potentials $\varphi_{R_{j}}^{o}$ are calculated using zero speed Green's function given in [34]. The actual difference between the linear strip-theory [15] and the blended method is because of the partial derivative term, $U \frac{\partial}{\partial x}$ present in the linearized dynamic pressure Eq. (20). In the blended method the partial derivative $U \frac{\partial}{\partial x}$ is performed explicitly, while in the linear strip theory of [15], this derivative is circumvented using an alternate form of Stoke's theorem as proposed by Ogilvie [35]. Ogilvie [36] suggested to evaluate the surface integral term $U \iint\left(\frac{\partial \varphi_{R_{j}}}{\partial x}\right) n_{i} d S$ of Eq. (26) as follows:

$U \iint\left(\frac{\partial \varphi_{R_{j}}}{\partial x}\right) n_{i} d S=-U \iint \varphi R_{j} m_{i} d S-U \int \varphi R_{j}[(t \times n) \cdot W] n_{i} d l$

The last term in Eq. (27) is a line integral over the boundary of mean position of the body at the intersection of the ship hull and the calm water line; where $t$ and $n$ are surface tangent and normal vectors respectively. 
Newman [33] showed that for a wall-sided ship $[(t \times n) \cdot W]=\frac{\partial \varphi_{s}}{\partial z} \cong 0$ at $z=0$, the vertical velocity due to the steady perturbation potential is of higher-order at the intersection of body and calm water line. This line integral is of higher-order and therefore may be neglected. Salvesen et al. [15] have also suggested to neglect the line integral along the water line and retaining the line integral of the end section only. There is experimental evidence also, which strongly suggest completely ignoring the line integral present in Eq. (27). If the steady perturbation velocity potential $\varphi_{s}=0$ then the line integral in Eq. (27) should be identically zero. In the linear part of the code developed for this study, the line integral shown in Eq. (27) has been completely neglected.

It may be recognized that the term $U \frac{\partial}{\partial x}$ has reduced from $W . \nabla$ due to setting $\varphi_{s}=0$ and linear approximations and decomposition of the full three-dimensional problem. Using the theorem given in appendix $\mathrm{A}$ of [35], it can be shown that $\iint W \cdot\left(\nabla \varphi_{R}\right) n d S \cong \iint \varphi_{R}(n \cdot \nabla) W d S$. The term $m_{\mathrm{i}}$ of Eq. (27) may be written in vector from as $\left(m_{1}, m_{2}, m_{3}\right)=-n \cdot \nabla W$ and $\left(m_{4}, m_{5}, m_{6}\right)=-n$. $\nabla(r \times W)=r \times\left(m_{1}, m_{2}, m_{3}\right)+W \times n$, where $r$ is the three-dimensional position vector. If the steady-perturbation velocity potential is neglected by setting $\varphi_{s}=0$ and let $U=1$, then from the above mentioned vector operations it can be shown that $m_{\mathrm{i}}=0$ for $\mathrm{i}=1,2,3,4$ and $m_{5}=n_{3}, m_{6}=$ $-n_{2}$. Eq. (26) can be rewritten in terms of $\varphi^{o} R_{j}, n_{i}, \varphi_{j}^{u}$ and $m_{i}$ for $i=1 \cdots 6$ and $j=1 \cdots 6$ as following:

$\omega_{e}^{2} A_{i j}-i \omega_{e} B_{i j}=-\rho i \omega_{e} \iint\left[\varphi^{o} R_{j} n_{i}+U\left(\varphi_{j}^{u} n_{i}-\frac{\varphi^{o} R_{j}}{i w_{e}} m_{i}\right)-\frac{U^{2}}{i w_{e}} \varphi_{j}^{u} m_{i}\right] d S$

As already defined the $\varphi^{u}=0$ for $j=1 \cdots 4, \varphi_{5}^{u}=\frac{\varphi_{3}}{i w_{e}}$ and $\varphi_{6}^{u}=-\frac{\varphi_{2}}{i w_{e}}$. Similarly $m=0$ for $j=1 \cdots 4, m_{5}=n_{3}$ and $m_{6}=-n_{2}$. The velocity potential $\varphi_{j}^{u}$ has resulted from body boundary condition in a rotating frame of reference. The term $m_{\mathrm{i}}$ is the consequence of replacing the integrand term $\widehat{U} \mathrm{i} \cdot \nabla\left(\varphi^{o} R_{j}+\varphi_{j}^{u}\right) n$ of Eq. (26) inside the vectorial surface integral of the linearized dynamic pressure given in Eq. (20) due to moving frame, with the term $\left(\varphi^{o} R_{j}+\varphi_{j}^{u}\right)(n \cdot \nabla) \widehat{U}$ i for surge, sway and heave modes. For roll, pitch and yaw modes, the integrand term $\widehat{U} \mathrm{i} \cdot \nabla\left(\varphi^{o} R_{j}+\varphi_{j}^{u}\right)(r \times n)$ is replaced with the term $\left(\varphi^{o} R_{j}+\varphi_{j}^{u}\right)[(r \times n \cdot \nabla) \widehat{U} \mathrm{i}+$ $\widehat{U} \mathrm{i} \times \mathrm{n}]$. Ogilvie et al. [35] showed mathematical validation for the interchangeability of the two terms inside a surface integral using the properties of velocity flow vector $\widehat{U}$ i. For the explicit evaluation of pressure one cannot take the advantage of interchangeability of these two terms. In the case of pressure, the partial derivative $U \frac{\partial}{\partial x}$ needs to be performed explicitly on the velocity potentials $\varphi^{o} R_{j}+\varphi_{j}^{u}$. The comparison between the radiation forces due to blended method and 
strip theory of [15] depends on how closely the numerical equivalence $\iint U \frac{\partial\left(\varphi^{o} R_{j}+\varphi_{j}^{u}\right)}{\partial x} n_{i} d S \cong$ $\iint U\left(\varphi_{j}+\varphi_{j}^{u}\right) m_{i} d S$ holds between the interchangeable two terms discussed above in the light of theorem given in [35].

$F_{j}=\int_{\frac{-L}{2}}^{\frac{L}{2}}\left[\int_{S(t)} P n_{j} d \delta\right] d x$

The blended scheme presented in this study focuses on explicit evaluation of dynamic pressures. The forces are then calculated by using a surface integral on the product of pressures and the body normal as shown in Equation 29. For the purpose of a comparison between the blended method and [15] strip-theory, Wigley-hull offsets were generated using Equation 30, where $B=2 m, \mathrm{~L}=20 m, \mathrm{~T}=-1 m$ are the beam, length and the draft of the ship respectively.

$Y=\frac{-B}{2}\left[1-0.95\left(\frac{X}{0.5 L}\right)^{2}\right]\left[1-\left(\frac{Z}{T}\right)^{2}\right]$

For the forced oscillation in sway mode, the comparison between linear and blended method is shown in Figure 4. It can be seen that sway into sway and sway into roll compare well, while sway into yaw does not. In sway into yaw mode, the linear complex force is significantly bigger than the radiation force predicted by blended method. In the [15] strip theory model, the cross coupling radiation force is coming from the term $i \omega_{e} U\left(\varphi_{j}^{u} n_{i}-\frac{\varphi R_{j}}{i \omega_{e}} m_{i}\right)$ of the Equation 28, for $i$ $=6, j=2$ this term reduces to $-U \varphi_{2} m_{6}$. This forward speed correction is only due to dynamic pressure in moving frame. Body boundary forward speed correction would be zero, because rotation of axis-system is zero. For the reduced term $-U \varphi R_{2} m_{6}=U \varphi R_{2} n_{2}$, effectively the partial derivative has been performed only on $n_{6}$. While in the blended method, the partial derivative $U \frac{\partial}{\partial x}$ is performed on $\varphi R_{2}$ and $n_{6}$ remains unchanged. Numerically, $\iint U \frac{\partial \varphi R_{2}}{\partial x} d S \neq$ $\iint U \varphi R_{2} n_{2} d S$. This explains the difference in sway into yaw mode shown in Figure 1 between linear and blended method.

For the forced heave oscillation, the comparison between linear and blended method is shown in Figure 5 and 6, respectively for resulting radiation and hydrostatic heave forces. The radiation and hydrostatic forces in all the modes compare well except for heave into pitch as in the linear model the partial derivative $U \frac{\partial}{\partial x}$ is performed only on $n_{5}$ instead of taking the partial derivative $U \frac{\partial}{\partial x}$ of $\varphi R_{3}$. 
Similarly the forced roll oscillation, comparison between linear and blended method is shown in Figure 7 and 8 respectively for resulting radiation and hydrostatic forces. In Figure 8, the roll and heave hydrostatic forces are not in good agreement. The Wigley-hull being non wall-sided is the reason for this small difference. When forced roll motion is given to a non-wall-sided body about a fixed axis, the vertical hydrostatic would not be in balance, unless the body is allowed to heave. In Figure 7, the radiation forces of roll into roll and roll into sway are in good agreement. However, in case of roll into yaw, the blended method has predicted lower radiation force. The forward speed correction due to the body boundary condition in rotating-frame of reference is zero because of $\varphi_{\mathrm{s}}=0$. In [15] strip theory, the only forward speed correction captured is due to linearized dynamic pressure in the moving coordinate. It can be shown again using similar reasoning already discussed for the case of sway into yaw radiation mode, that the partial derivative $U \frac{\partial}{\partial x}$ is performed only on $n_{6}$ instead of taking the partial derivative $U \frac{\partial}{\partial x}$ of $\varphi_{4}$ in [15] strip theory.

In Figure 9 and 10, the comparison between linear and blended method is shown for resulting radiation and hydrostatic forces respectively for the forced pitch oscillation. In Figure 10, the pitch into heave force is not in good agreement. The reason for this vertical unbalanced hydrostatic is the non-wall-sided hull form is forced to pitch about a fixed axis without allowing to heave. This vertical unbalanced hydrostatic will vanish if the ship is allowed to heave freely. In Figure 9 the radiation force pitch into pitch and pitch into surge are in good agreement. But the blended method has predicted higher force in pitch into heave mode. In the case of the linear model, the cross coupling radiation force is a result of the term $i \omega_{e} U\left(\varphi_{j}^{u} n_{i}-\frac{\varphi R_{j}}{i \omega_{e}} m_{i}\right)$ of the Equation 28. For $i=$ $3, j=5$ this term reduces to $i \omega_{e} U \varphi_{5}^{u} n_{3}=U \varphi R_{3} n_{3}$. This forward speed correction is coming from the body boundary condition in the rotating axis-system. It is observed that the forward speed correction arising from dynamic-pressure due to moving frame of reference could not be captured appropriately in [15] linear strip-theory model. However, the blended method is adequately taking care of both the forward speed corrections arising from the rotating body boundary condition and dynamic pressure in moving frame of reference. This is the reason that the blended method is predicting higher pitch into heave radiation force than [15] linear strip theory.

For the forced yaw oscillation in Figure 11, the comparison is shown between the linear strip theory and blended method. The resulting radiation forces for the modes yaw into sway and yaw into roll are not in good agreement, the blended method predicts higher forces. The reason for these higher cross coupling terms can be explained based on the argument presented in the previous paragraph for the pitch into heave mode. In linear strip theory model, the forward speed corrections resulting from dynamic-pressure in moving frame have been almost neglected in yaw into roll and yaw into sway modes. The blended method by comparison, partially accounts for the forward speed corrections in the moving frame after setting $\varphi_{\mathrm{s}}=0$. 


\subsection{Blended Method for Diffraction and Froude-Kriloff Forces in Moving Frame}

The scattered or diffracted velocity potential $\varphi_{D}$ is obtained by solving the Laplace Equation 31 with the free surface and body boundary conditions 22 and 33. The scattering phenomenon may be described as the restrained body in the steady moving frame with the velocity $U \neq 0$ or in the earth fixed coordinate with the velocity $U \equiv 0$ exposed to incident waves. There is no rotation of the axis-system; the forward speed correction arising in the body boundary conditions should vanish. This can be seen from Equation 34, where the term $[(W \cdot \nabla) X] \cdot n$ vanishes explicitly for $X=0$ or for the non-rotating body. The nonlinear body boundary condition for the scattered velocity potential including the steady perturbation potential would be as follows:

$\nabla^{2} \Phi(x, y, z, t)=0$

$\frac{D}{D t} S(x, y, z, t)=0$ at $z=\zeta$

where $\nabla^{2}$ is Laplace operator and $\mathrm{D}$ is the total / cumulative derivative.

$$
\begin{aligned}
& \frac{\partial \varphi D}{\partial n}=\nabla \varphi D \cdot n=-\nabla \varphi I \cdot n \\
& \frac{\partial \varphi D}{\partial n}=\nabla \varphi D \cdot n=-\nabla \varphi I \cdot n+[(W \cdot \nabla) X-(X \cdot \nabla) W] \cdot n
\end{aligned}
$$

In the steady moving frame, the terms involving $\mathrm{W}$ and $\mathrm{X}$ would be identically zero if $\varphi_{s}$ is neglected. Effectively the Equation 34 reduces to the Equation 33. For the diffraction problem in a moving frame of reference, the only forward speed correction would be from dynamic-pressure Equation 10 or its linearized version in Equation 20 after substituting $\varphi_{D}$ for $\varphi_{R}$. For the blended method, the diffraction force is as follows:

$F_{j}^{D}=-\rho \iint\left[\frac{\partial \varphi D}{\partial t}+W \cdot \nabla \varphi D+\frac{1}{2} \nabla \varphi D \cdot \nabla \varphi D\right] n_{j} d S$

The surface integral in Equation 35 is for the instantaneous submerged body. If the steady perturbation velocity potential is neglected $\varphi_{S}$, the term $W \cdot \nabla \varphi D$ in Equation 35, may be simplified as $-\widehat{U} i \cdot \nabla \varphi D=-U \frac{\partial \varphi D}{\partial x}$. In the blended method, the partial derivative $-U \frac{\partial}{\partial x}$ is performed numerically using radial basis function. 
$F_{D_{j}}=\rho \int_{L / 2}^{L / 2}\left[\int_{S_{o}} \nabla \varphi I \cdot n_{j} \varphi R d \delta\right] d x$

In case of the linear strip-theory model, the scattering velocity potential is not known explicitly. The linear diffraction forces are calculated from the incident and radiation velocity potentials after applying Green's second identity on the surface integral involving all the bounding surfaces of the linear boundary value problem, as shown in Haskind-relation 36 for zero forward speed. For the problem involving forward speed, one may assume that the speed dependent terms in the linearized free surface boundary condition are of higher-order and therefore could be neglected. The linear approximation of Equation 35 may be rewritten as:

$F_{j}^{D}=-\rho \iint\left[i \omega_{e} \varphi D-\widehat{U} i \cdot \nabla \varphi D\right] n_{j} d S$

$\nabla \varphi(x, y, z, t) \cdot n=V \cdot n \quad$ at $S_{o}$

where $\mathrm{V}$ denotes ships unsteady oscillatory velocity in the moving frame.

Considering body boundary condition 38, the first term $\left[i \omega_{e} \varphi D\right] n_{j}$ of Equation 37 can be replaced by $\left(-\nabla \varphi R_{j} \cdot n\right) \varphi D$. The equality $\nabla \varphi R_{j} \cdot n \varphi D=\nabla \varphi D \cdot n \varphi R_{j}$ for the integrand of surface integral holds because of the second identity of Green's theorem applied to all the bounding surface of the boundary value problem. From the body boundary condition 33 of the scattering problem $\nabla \varphi D \cdot n=-\nabla \varphi I \cdot n$, where $\varphi I$ is incident wave velocity potential defined in Equation 39. The first term $\left[i \omega_{e} \varphi D\right] n_{j}$ in Equation 37 represents exactly the Haskind-relation of Equation 36 for zero speed.

$\varphi I=\frac{i g \propto}{\omega} e^{k z} e^{-i k(x \cos \beta+y \sin \beta)} e^{i \omega_{e} t} \quad$ at $z=\zeta$

where $k=2 \pi / \lambda$ is the wave number and $\beta$ is the heading angle.

The second term of Equation 37 may be written in vector notation as $[-\widehat{U} i \cdot \nabla \varphi D] n$. The equality $\iint[-\widehat{U} i \cdot \nabla \varphi D] n d S=\iint[\varphi D(n \cdot \nabla)](-\widehat{U} i) d S$ can be shown by using properties of flow vector $W$ and theorem given in appendix A of [35]. The term $[\varphi D(n \cdot \nabla)](-\widehat{U} i)$ is identically zero for surge sway and heave modes of motion. For roll, pitch and yaw, the second term of Equation 37 may be written in vector form as $[-\widehat{U} i \cdot \nabla \varphi D](r \times n)$. Again, using [15] theorem, one can show the equivalence $\iint[-\widehat{U} i \cdot \nabla \varphi D](r \times n) d S=\iint \varphi D[((r \times n)$. $\nabla)(-\widehat{U} i)+(n \times-\widehat{U} i)] d S$, which is zero for roll, and in pitch and yaw modes are $U \varphi D n_{3}=$ $-U \varphi D n_{2}$ respectively. 
Using body boundary condition 33, and Green's second identity for surface integral over all the linearized bounding surfaces, the forward speed correction can be written in terms of radiation and incident velocity potentials for pitch and yaw as $\frac{U}{i \omega_{e}}(\nabla \varphi I \cdot n) \varphi R_{3}$ and $-\frac{U}{i \omega_{e}}(\nabla \varphi I \cdot n) \varphi R_{2}$, respectively. These are the same forward speed corrections as given by [15] derived from the body boundary condition due to rotation of axis-system. The forward speed corrections derived here are arising from dynamic pressure in moving frame of reference.

Similarly, the Froude-Kriloff forward speed corrections are also from the dynamic-pressure due to the moving frame. It can be derived analytically from incident velocity potential as given in Equation 39. The comparison between blended and [15] linear strip-theories for Froude-Kriloff and diffraction forces are shown in the Figures 12 and 13 respectively. The Froude-Kriloff forces compare well in all six modes. However, the diffraction forces are significantly different for the pitch and yaw modes. The reason for the difference is the numerical inequality between the term $-U \frac{\partial \varphi D}{\partial x}$ and the terms used in the pitch and yaw modes $\frac{U}{i \omega_{e}}(\varphi I \cdot n) \varphi R_{3}$ and $-\frac{U}{i \omega_{e}}(\varphi I \cdot n) \varphi R_{2}$ respectively.

\subsection{Comparison Between Blended Method and Experimental Data}

As shown in the previous two sections, the cross coupling terms for radiation forces resulting from the blended method developed in this study, do not compare well with Ogilvie [35] and Salvesen [15] results. Similarly, diffraction forces in pitch and yaw modes also shows disagreement with Ogilvie [35] and Salvesen [15] findings. In this section the blended method for the forward speed correction is compared with the available experimental data.

Journee [37] conducted experiments on four different Wigley-hull forms for three different ship speeds corresponding to Froude numbers $F_{n}=0.2, F_{n}=0.3$ and $F_{n}=0.4$ in head seas. The author also gave his judgement on reliability of experimental data. A comparison between blended method and experimental data for Wigley-hull-III for the radiation and wave loads in head seas has been demonstrated in this section. For the forced oscillations, the experimental data is given in terms of added-mass and damping coefficients. For the purpose of comparison with the blended method, a complex radiation force time series is generated using the relationship $\widetilde{F_{R}}(t)=$ $\left(\omega_{e}^{2} A-i \omega_{e} B\right) e^{i \omega_{e} t}$. Similarly, the wave load data is given as normalized amplitude and phase, and a complex wave load force series is generated using the relationship $\widetilde{F_{D}}(t)=|F| e^{i\left(\omega_{e} t+\theta\right)}$. The offsets for the Wigley-hull-III are obtained using Equation 40 as given in [37]. 
$Y=-\frac{B}{2}\left[1-\left(\frac{X}{0.5 L}\right)^{2}\right]\left[1-\left(\frac{Z}{T}\right)^{2}\right]\left[1+0.2\left(\frac{Z}{T}\right)^{2}\right]$

Radiation and wave loads calculated by the blended method are in good agreement with the experimental data given in [37], for the various forward speeds. For the forced heave motion given to Wigley-hull-III moving with Froude number $F_{n}=.2$, the resulting radiation forces are comparing well as shown in Figure 14. A similar comparison for the forced pitch motion and Froude number $F_{n}=.4$ is shown in Figure 16. The cross coupling terms obtained from the blended method developed in the present research and experiments by Journee [37] are in good match as seen in Figures 14 and 16. The comparison between linear and blended method, for the accompanying hydrostatic forces is shown in Figures 15 and 17. A good agreement between the blended method and experiments by Journee [37] for the wave loads with forward speed corresponding to Froude number $F_{n}=.3$ is found as shown in Figure 18. The wave loads shown in Figure 18 are the sum of diffraction, Froude-Kriloff and hydrostatic forces in the head seas. In the wave loads, the FroudeKriloff is the dominating force, the diffraction and hydrostatic forces are approximately 20 and 1 percent of the total wave loads as found numerically using the blended method.

It may be noticed that in case of cross coupling terms, the blended method is comparing well with the experimental data and not with the results obtained using slender body forward speed corrections suggested by Ogilvie et al. [35]. To find the reason for this somewhat surprising outcome, one needs to explore the underlying assumptions, conditions and properties of different functions used in the derivation to prove the equality of the integrals $\iint \widehat{U} i \cdot \nabla\left(\varphi_{R_{j}}^{o}+\varphi_{j}^{u}\right)(r \times$ $n) d S=\iint\left(\varphi_{R_{j}}^{o}+\varphi_{j}^{u}\right)[(r \times n \cdot \nabla) \widehat{U} i+\widehat{U} i \times n] d S$ of a surface integral by Ogilvie et al. [35]. This equality does not hold in general for all surface integrals or their integrands.

\section{Discussion}

For the mode, heave into heave, the first component of the forward speed correction is evidently zero. After integrating along the ship length, the net effect of the second component of the forward speed correction $U \frac{\partial_{\varphi R_{3}}}{\partial x}$ also becomes zero. The velocity potential $\varphi R_{3}$ is an even function with respect to $x$, its derivative $\frac{\partial_{\varphi R_{3}}}{\partial x}$ would be an odd function. The $n_{3}$, a component of the normal $n$ is an even function with respect to $x$. The product $\frac{\partial_{\varphi R_{3}}}{\partial x} n_{3}$ is an odd function with respect to $x$. Therefore its integration along the longitudinal axis of the ship is zero. Effectively, there is no forward speed correction in the mode heave into heave. The slender-body theory, experiments and 
the blended method are all in good agreement. However in the blended method, the derivative $\frac{\partial_{\varphi R_{3}}}{\partial x}$ is calculated to predict the nodal pressure inclusive of the forward speed effects. After integration of the pressures along the ship length, the forward speed effects vanish.

In case of pitch into heave, the forward speed correction comprises both of the components. In the blended method, the integrand of this correction may be expressed as the product $U\left(\frac{\partial_{\varphi R_{5}}}{\partial x}+\right.$ $\left.\frac{\partial_{\varphi R_{3}}}{\partial x}\right) n_{3} . \varphi R_{3}$ is the correction due to body boundary condition and the derivative with respect to $x$ accounts for the evaluation of pressure in the moving frame. The second term of the integrand $\frac{\partial_{\varphi R_{3}}}{\partial x} n_{3}$ is an odd function with respect to $x$, the net effect vanishes after integrating along the ship length. Thus in the blended method, the net effective forward speed correction is proportional to the surface integral of the term $\frac{\partial_{\varphi R_{5}}}{\partial x} n_{3}$. By assuming $\varphi R_{5} \approx-x \varphi R_{3}(y, z)$, one can show that this is identically equal to $-\varphi R_{3} n_{3}$, which is the same as suggested by slender body theory. This approximation holds only if $\varphi R_{3}$ is assumed to be the function $y, z$ only. There is no explicit or implicit dependence on $x$, while this is not the case in the blended method and in the real physics of the problem. The two-dimensional $\varphi R_{3}(y, z)$ is transformed into its corresponding threedimensional quantity by using an appropriate radial basis function. Then the derivative $\frac{\partial_{\varphi R_{3}(x, y, z)}}{\partial x} n_{3}$ is calculated on a three-dimensional quantity. This approach is closer to the real physics of the problem. That is why the results from the blended method are in good agreement with the experimental results.

ISSC [38] and Finn et al. [39] have shown comparisons of vertical bending moment on SR175 container ship with various numerical calculations and experimental data. Their results are reproduced using blended method and are superimposed on them as shown in Figure 19. It is observed that the maximum sagging moment is approximately twice the value of maximum hogging moment. These results are obtained by approximating the sectional weight distribution with the corresponding sectional buoyancy forces. The results from the blended method are comparing well with the results of other researchers.

\section{Conclusions}

In this paper the difference between the forward speed correction used for time simulation in the blended method and the strip-theory presented by Salvesen et al. [15] in the frequency domain has been explained. Using the previous work of Newman [33] and Ogilvie et al. [35], a basis is developed for the comparison between the blended method and the frequency domain linear 
model. The calculated results for the steady and unsteady problems are compared with published model test results. Good agreement is obtained, which validates the present numerical method. The comparison of the calculated results with the experimental data shows that the blended method is effective and feasible. The present method can be further extended to dynamic structural design and maneuvering problems.

\section{References}

[1] R. Yousefi, R. Shafaghat, and M. Shakeri, "Hydrodynamic Analysis Techniques for Highspeed Planing Hulls,” Appl. Ocean Res., vol. 42, pp. 105-113, Aug. 2013.

[2] L. . Doctors, "Representation of Planing Surfaces by Finite Pressure Elements," in 5th Australian Conference on Hydraulics and Fluid Mechanics., 1974.

[3] X. Wang and A. H. Day, "Numerical instability in linearized planing problems," Int. J. Numer. Methods Eng., vol. 70, no. 7, pp. 840-875, May 2007.

[4] C. Lai and A. W. Troesch, "A Vortex Lattice Method for Hight-Speed Planning," Int. J. Numer. Methods Fluids, vol. 22, no. 6, pp. 495-513, Mar. 1996.

[5] K. Benedict, N. Kornev, M. Meyer, and J. Ebert, "Complex mathematical model of the WIG motion including the take-off mode," Ocean Eng., vol. 29, no. 3, pp. 315-357, Mar. 2002.

[6] Z. Zhou, "A Theory and Analysis of Planing Catamarans in Calm and Rough Water," University of New Orleans, United States, 2003.

[7] M. Kandasamy et al., "CFD validation studies for a high-speed foil-assisted semi-planing catamaran,” J. Mar. Sci. Technol., vol. 16, no. 2, pp. 157-167, Jun. 2011.

[8] R. Yousefi, R. Shafaghat, and M. Shakeri, "High-speed planing hull drag reduction using tunnels," Ocean Eng., vol. 84, pp. 54-60, Jul. 2014.

[9] Y. Jiang, H. Sun, J. Zou, A. Hu, and J. Yang, "Analysis of tunnel hydrodynamic characteristics for planing trimaran by model tests and numerical simulations," Ocean Eng., vol. 113, pp. 101-110, Feb. 2016.

[10] M. Ghassabzadeh and H. Ghassemi, "Determining of the hydrodynamic forces on the multi-hull tunnel vessel in steady motion," J. Brazilian Soc. Mech. Sci. Eng., vol. 36, no. 4, pp. 697-708, Oct. 2014.

[11] S. E. Hirdaris et al., "Loads for Use in the Design of Ships and Offshore Structures," Ocean Eng., vol. 78, pp. 131-174, Mar. 2014.

[12] P. Finn., "Large Amplitude Nonlinear Seakeeping Using a Desingularized Method - PhD thesis," University of Michigan, 2003.

[13] C. P. Kent, "A Pseudo-Spectral Method for Calculating Wave-Body Interaction Using an Explicit FreeSurface Formulation - PhD thesis," University of Michigan, 2005.

[14] O. Faltinsen, "A Rational Strip Theory of Ship Motions Part II - PhD thesis," University 
of Michigan, 1971.

[15] N. Salvesen, E. O. Tuck, and O. Faltinsen, "Ship Motions and Sea Loads," Trans. Soc. Nav. Archit. Mar. Eng., vol. 78, pp. 250-287, 1970.

[16] O. M. Faltinsen, Hydrodynamics of high-speed marine vehicles. New York: Cambridge University Press, 2005.

[17] B. R. Savander, S. M. Scorpio, and R. K. Taylor, "Steady hydrodynamic of planing surface.," J. Sh. Res., vol. 46, pp. 248-279, 2002.

[18] H. Ghassemi and M. Ghiasi, "A combined method for the hydrodynamic characteristics of planing crafts," Ocean Eng., vol. 35, no. 3-4, pp. 310-322, Mar. 2008.

[19] H. Ghassemi and S. Yu-min, "Determining the hydrodynamic forces on a planing hull in steady motion," J. Mar. Sci. Appl., vol. 7, no. 3, pp. 147-156, Sep. 2008.

[20] A. Reza Kohansal and H. Ghassemi, "A numerical modeling of hydrodynamic characteristics of various planing hull forms," Ocean Eng., vol. 37, no. 5-6, pp. 498-510, Apr. 2010.

[21] H. Kihara, S. Naito, and M. Sueyoshi, "Numerical analysis of the influence of abovewater bow form on added resistance using nonlinear slender body theory," Sh. Res., vol. 49, pp. 191-206, 2005.

[22] G. Giorgi and J. V. Ringwood, "Analytical representation of nonlinear Froude-Krylov forces for 3-DoF point absorbing wave energy devices," Ocean Eng., vol. 164, pp. 749759, Sep. 2018.

[23] Z.-M. Yuan, A. Incecik, and D. Alexander, "Verification of a new radiation condition for two ships advancing in waves," Appl. Ocean Res., vol. 48, pp. 186-201, Oct. 2014.

[24] Z.-M. Yuan, A. Incecik, S. Dai, D. Alexander, C.-Y. Ji, and X. Zhang, "Hydrodynamic interactions between two ships travelling or stationary in shallow waters," Ocean Eng., vol. 108, pp. 620-635, Nov. 2015.

[25] S. Rajendran, N. Fonseca, and C. Guedes Soares, "Effect of surge motion on the vertical responses of ships in waves," Ocean Eng., vol. 96, pp. 125-138, Mar. 2015.

[26] S. Rajendran, N. Fonseca, and C. Guedes Soares, "Prediction of vertical responses of a container ship in abnormal waves," Ocean Eng., vol. 119, pp. 165-180, Jun. 2016.

[27] M. S. Khalid, S. Nisar, and A. Troesch, "Radiation and Diffraction Velocity Potentials for Multi-hulled Vessels in 2-D," Arab. J. Sci. Eng., vol. 41, no. 11, pp. 4573-4582, Nov. 2016.

[28] G. B. Wright and B. Fornberg, "Scattered node compact finite difference-type formulas generated from radial basis functions," J. Comput. Phys., vol. 212, no. 1, pp. 99-123, Feb. 2006.

[29] J. C. Carr, R. K. Beatson, B. C. McCallum, W. R. Fright, T. J. McLennan, and T. J. Mitchell, "Smooth surface reconstruction from noisy range data," in Proceedings of the 1 st international conference on Computer graphics and interactive techniques in Austalasia and South East Asia - GRAPHITE '03, 2003, p. 119.

[30] J. C. Carr et al., "Reconstruction and representation of 3D objects with radial basis 
functions," in Proceedings of the 28th annual conference on Computer graphics and interactive techniques - SIGGRAPH '01, 2001, pp. 67-76.

[31] A. I. Tolstykh and D. A. Shirobokov, "On using radial basis functions in a ?finite difference mode? with applications to elasticity problems," Comput. Mech., vol. 33, no. 1, pp. 68-79, Dec. 2003.

[32] M. S. Khalid, "Simulation of Euler-Equations of Motion and Blended-Nonlinear Hydrodynamics for Multi-Hulled Vessels - PhD thesis," University of Michigan, 2007.

[33] J. N. Newman, "The Theory of Ship Motions," Adv. Appl. Mech., pp. 221-283, 1979.

[34] W. Frank, "Oscillation of cylinder in or below the free surface of deep fluids - Technical report," 1967.

[35] T. F. Ogilvie and E. O. Tuck, "A Rational Strip Theory of Ship Motions Part - PhD thesis," 1969.

[36] T. F. Ogilvie, "Singular-Perturbation Problems in Ship Hydrodynamics," in Advances in Applied Mechanic, 1977, pp. 91-188.

[37] I. J and M. J. Journee, "Experimental and calculations on four wigley hull form. Technical Report 909," 1992.

[38] ISSC., "Extreme hull girder loading by Special task committee vi.1," 14th Int. Sh. Offshore Struct. Congr., vol. 2, pp. 263-320, 2000.

[39] P. Finn, R. F. Beck, A. W. Troesch, and Y. S. Shin, "Nonlinear impact loading in an oblique seaway," in 20th International Conference On Offshore Mechanics and Arctic Engineering, 2001. 


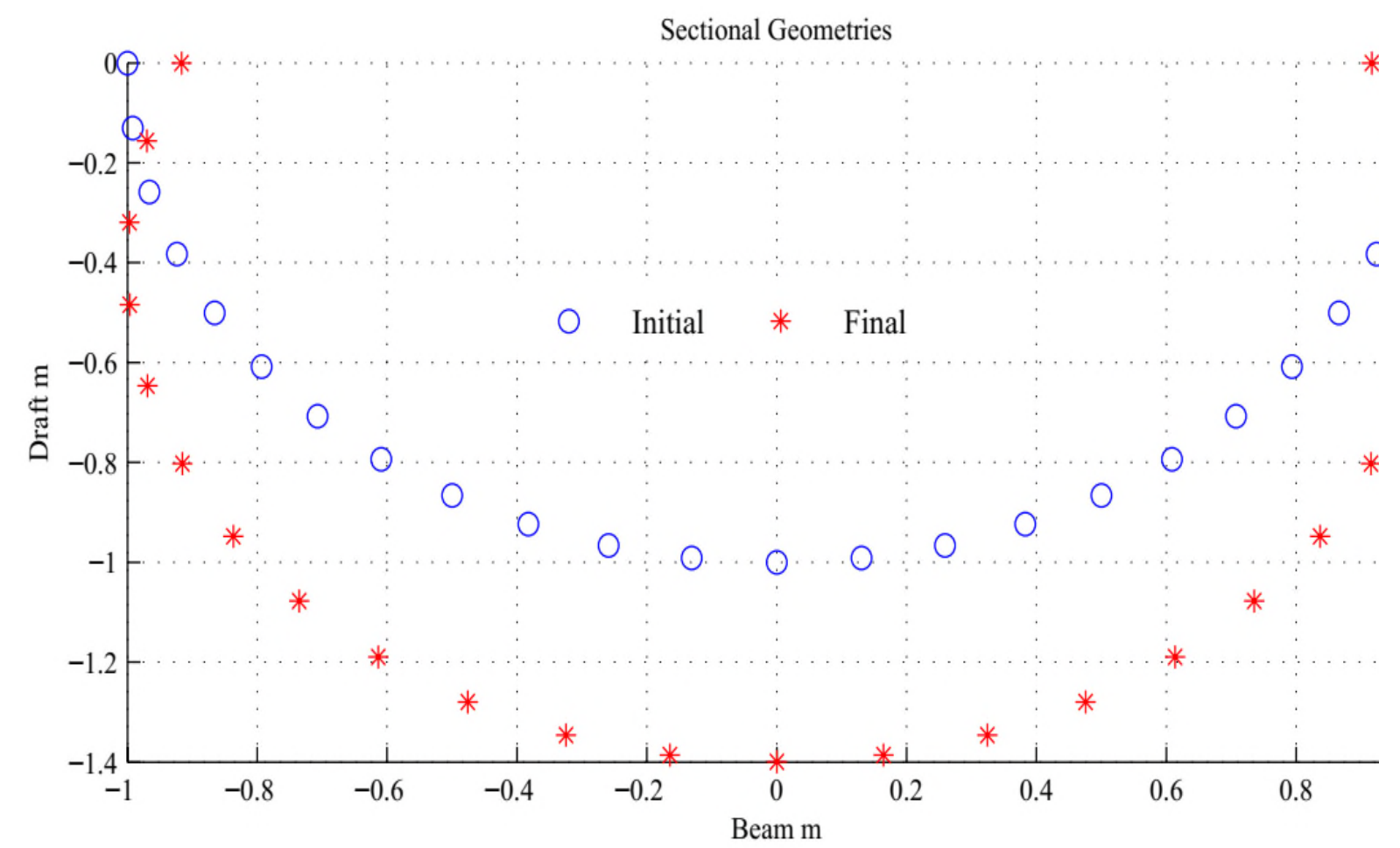

Figure 1: Two circular-sections, $\lambda_{i}$ based on the original body-section to estimate the function corresponding to changed or final body-section, submergence $=.4 R$ 

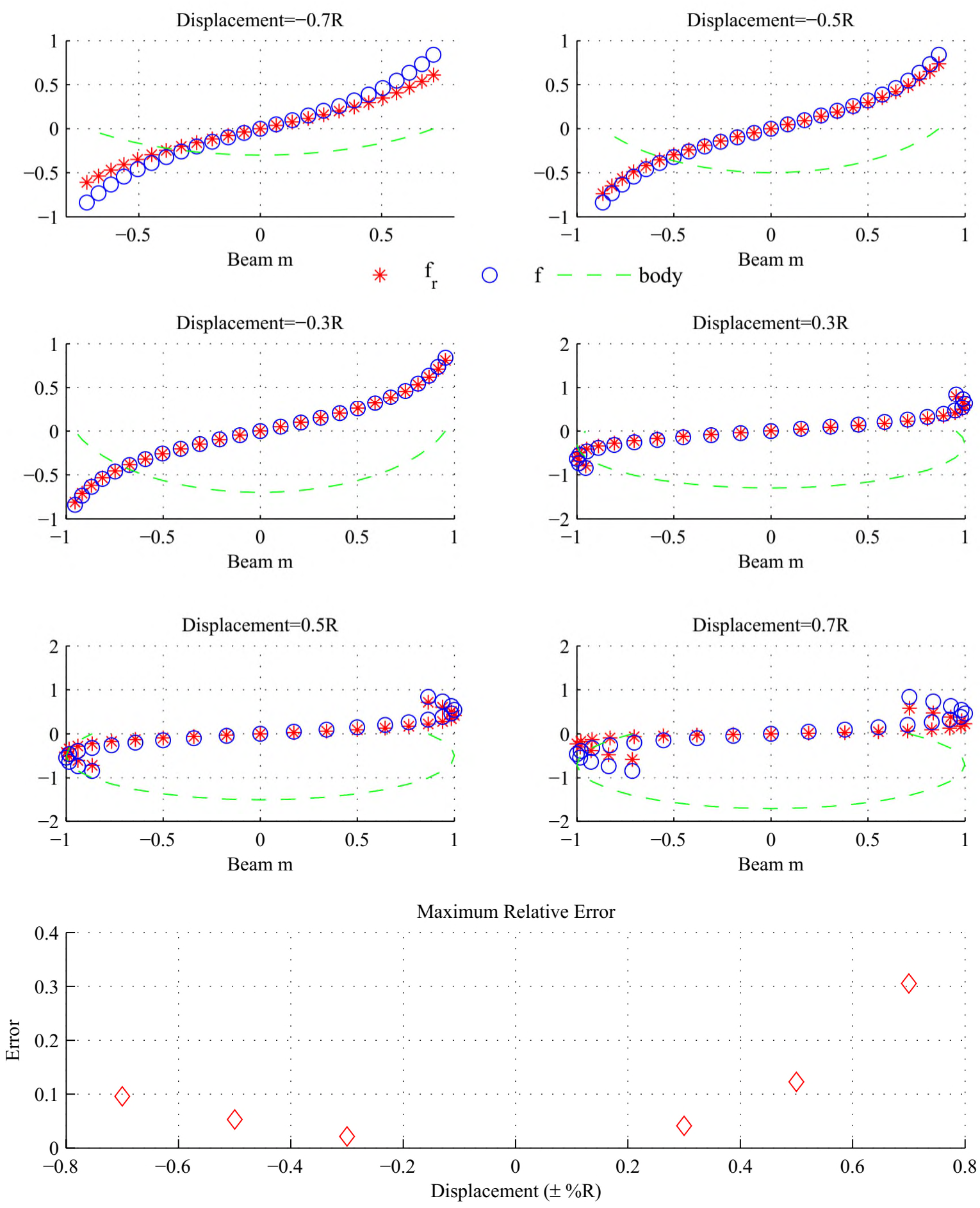

Figure 2: Comparing nodal values of an exact function $f$ vs estimated function $f_{r}$ on a varying cylindrical section 

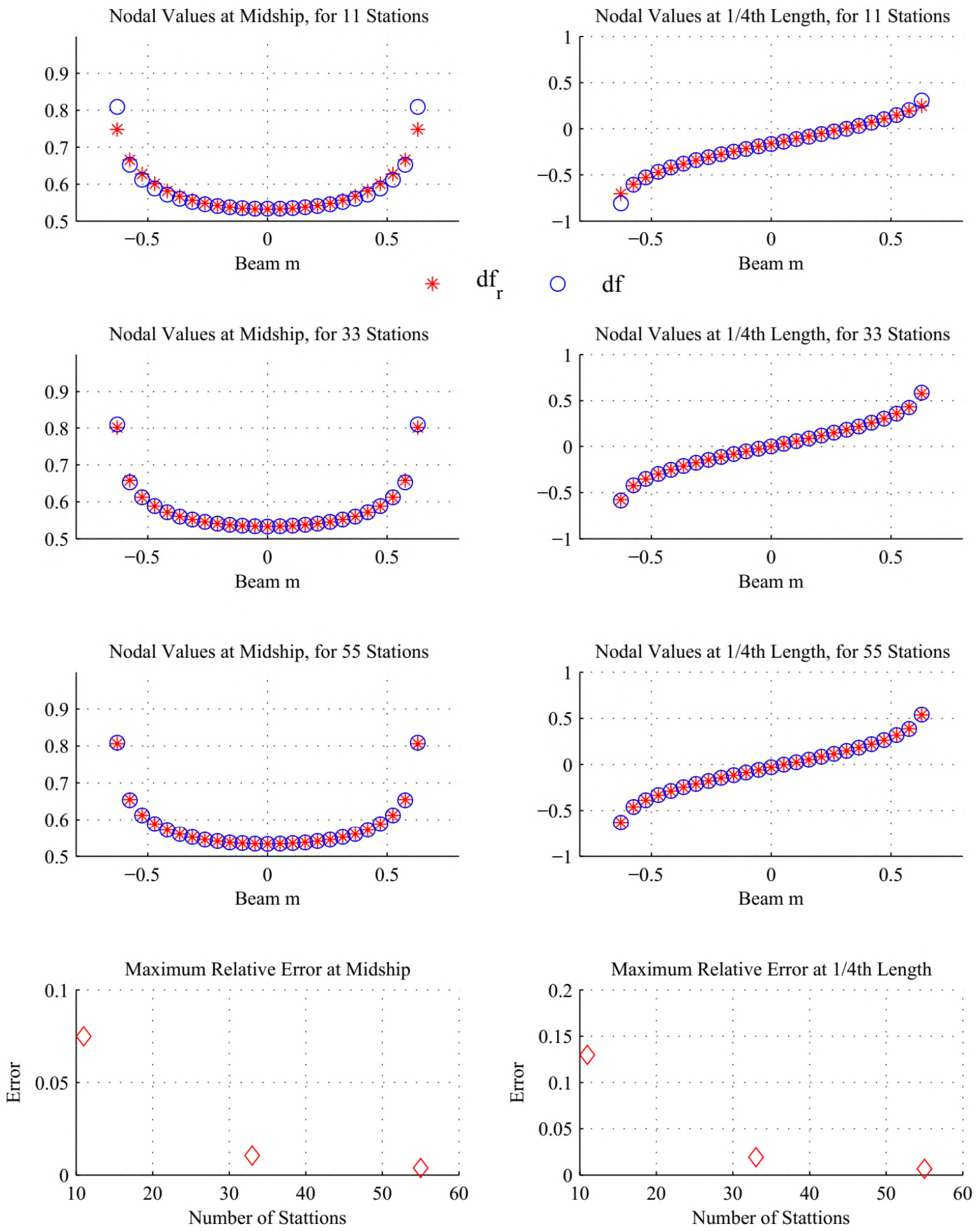

Figure 3: Comparing nodal values of an exact function $d f=\frac{\partial f(x, y, z)}{\partial x}$ vs. estimated function $d f r=\frac{\partial f_{r}(x, y, z)}{\partial x}$ on a cylinder varying the number of station along length 

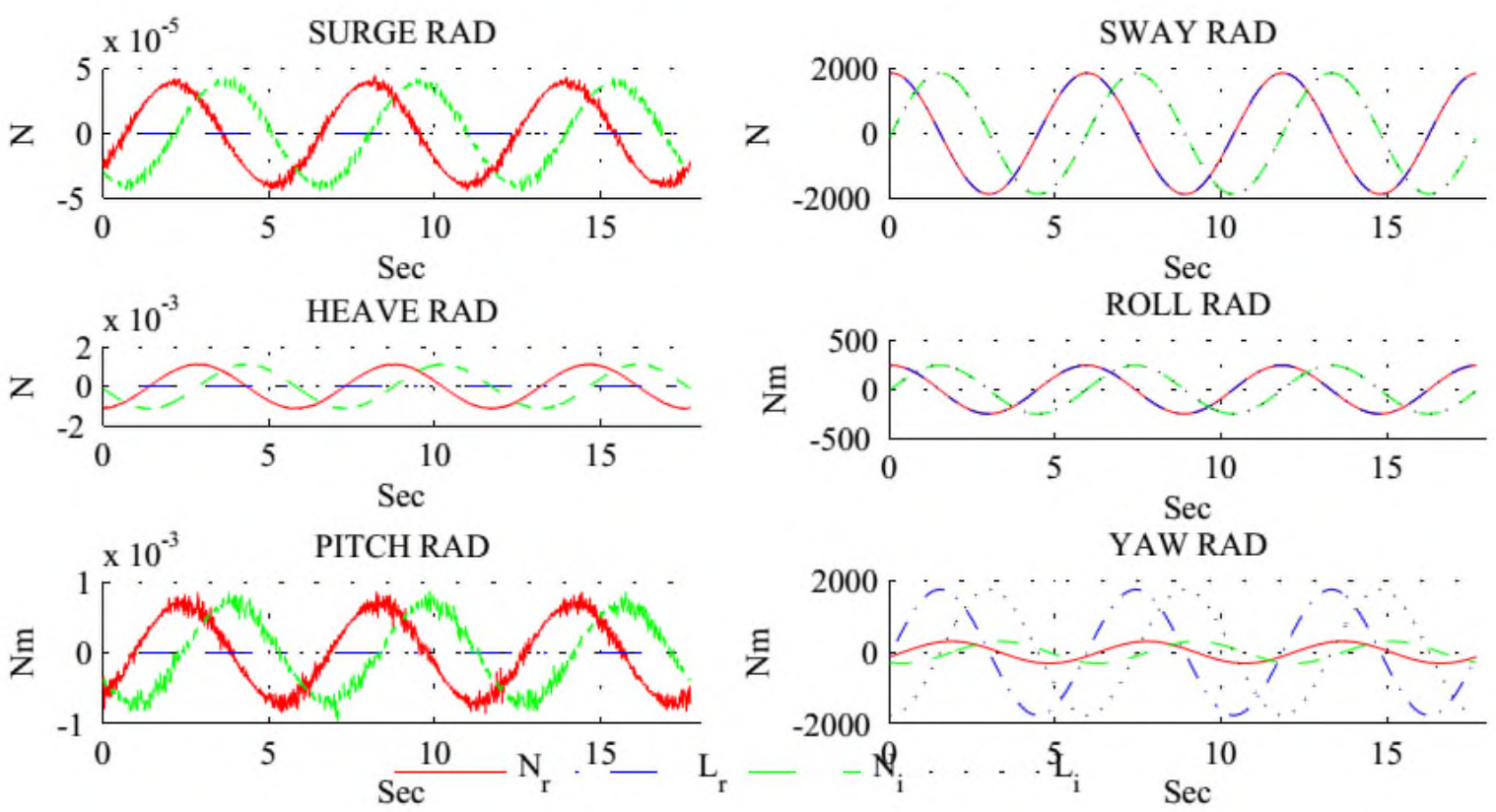

Figure 4: $0.05 m$ Forced sway radiation, linear $L$ vs quasi-nonlinear $N, \omega_{e}=1.066 \mathrm{rad} / \mathrm{sec}, V=$ $1 \mathrm{~m} / \mathrm{sec}, \mathrm{Fn}=0.0714$, Wigley-hull
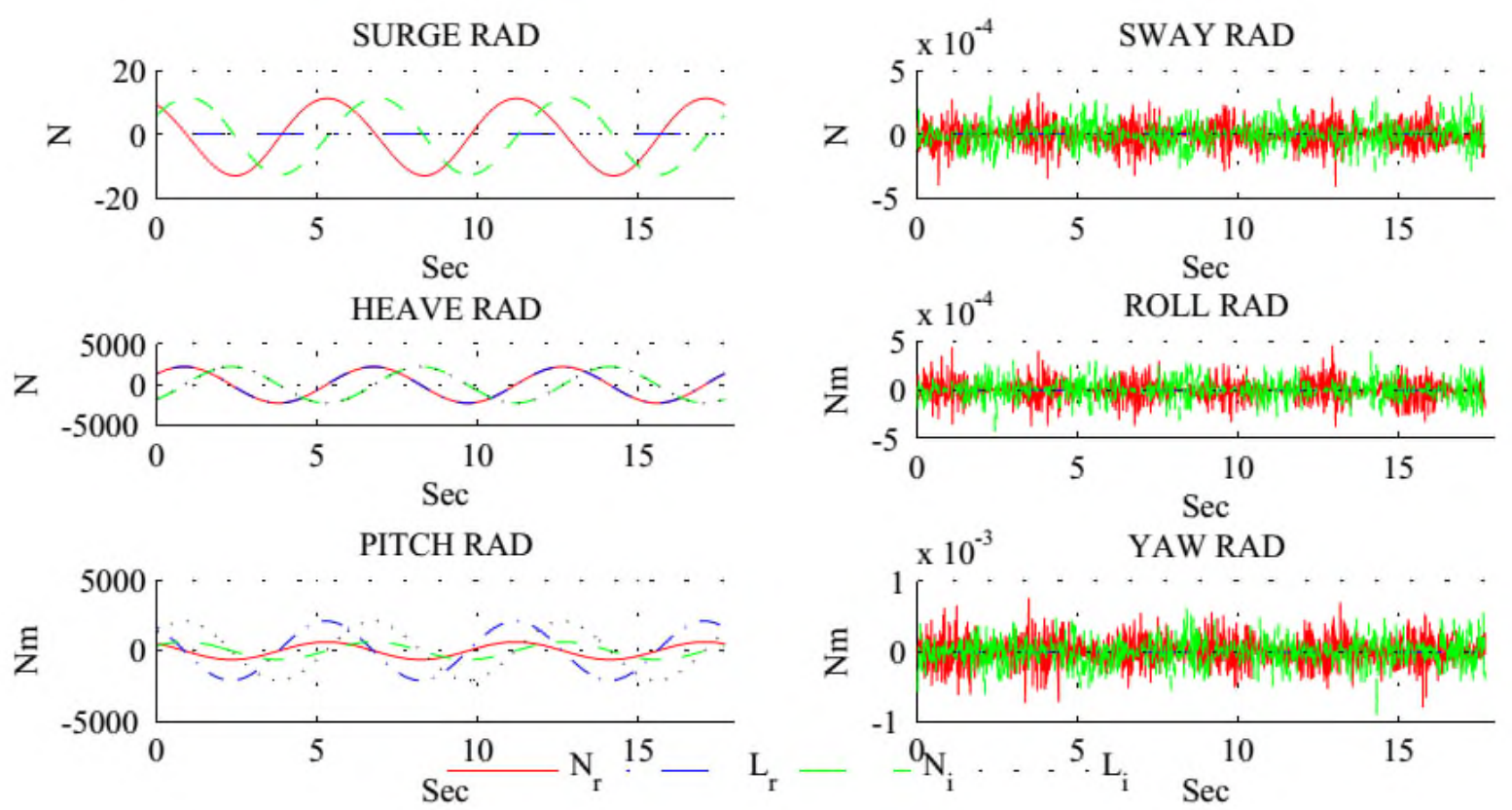

Figure 5: $0.05 \mathrm{~m}$ Forced heave radiation, linear $L$ vs quasi-nonlinear $N, \omega_{e}=1.066 \mathrm{rad} / \mathrm{sec}, \mathrm{V}=$ $1 \mathrm{~m} / \mathrm{sec}, \mathrm{Fn}=0.0714$, Wigley-hull 
SURGE HYDROSTATICS
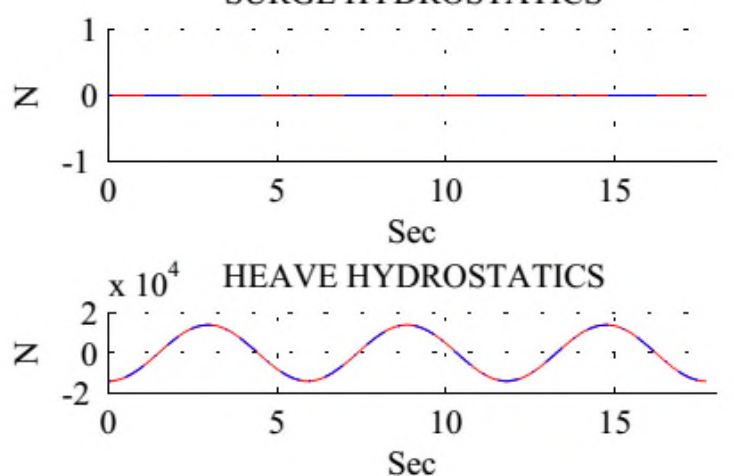

PITCH HYDROSTATICS

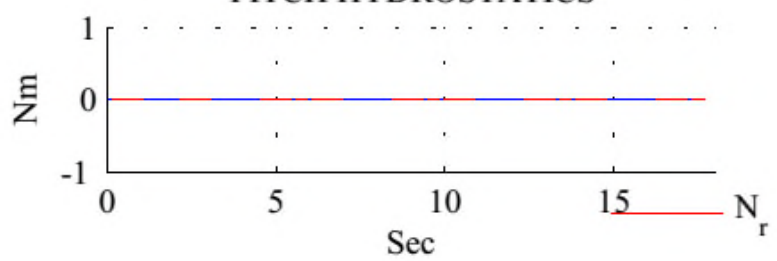

SWAY HYDROSTATICS

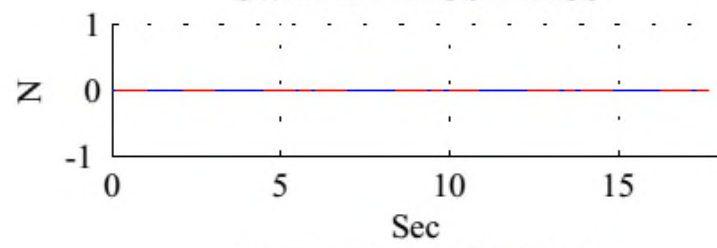

ROLL HYDROSTATICS

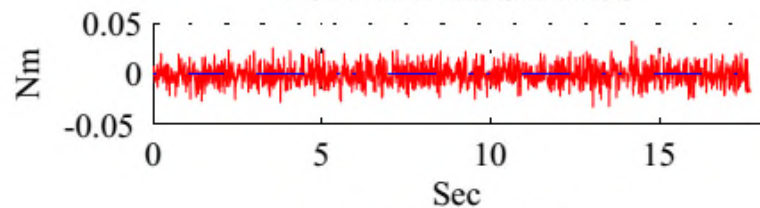

YAW HYDROSTATICS

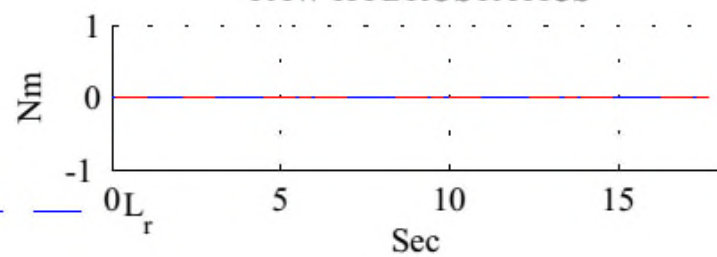

Figure 6: $0.05 m$ Forced heave hydrostatic, linear $L$ vs quasi-nonlinear $N, \omega_{e}=1.066 \mathrm{rad} / \mathrm{sec}$, $V=1 \mathrm{~m} / \mathrm{sec}, \mathrm{Fn}=0.0714$, Wigley-hull

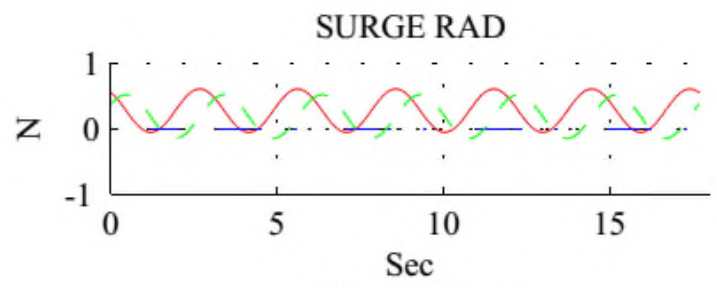

HEAVE RAD

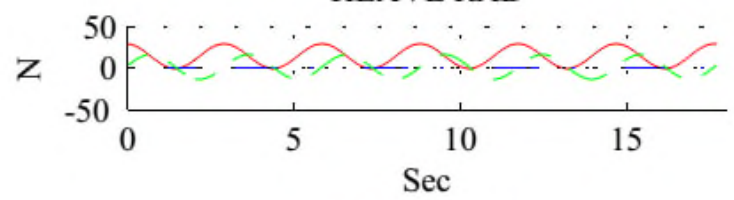

PITCH RAD

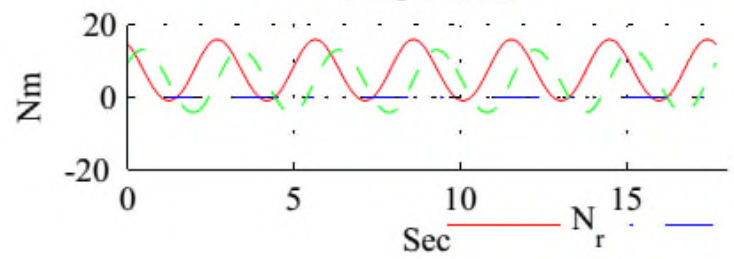

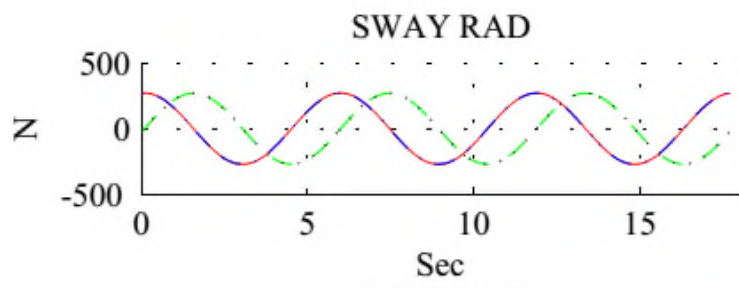

ROLL RAD

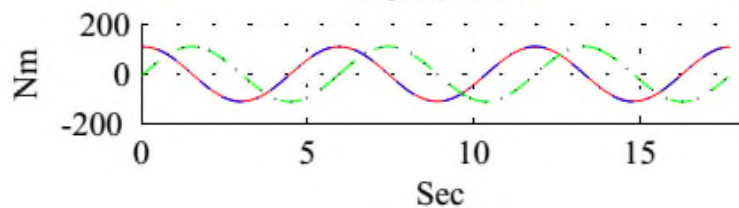

YAW RAD

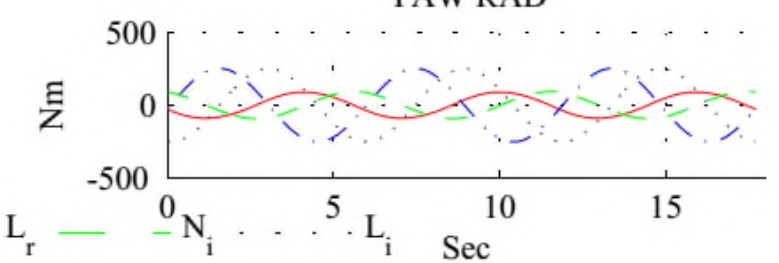

Figure 7: $0.05 \mathrm{rad}$ Forced roll radiation, linear $L$ vs quasi-nonlinear $N, \omega_{e}=1.066 \mathrm{rad} / \mathrm{sec}, \mathrm{V}=$ $1 \mathrm{~m} / \mathrm{sec}, \mathrm{Fn}=0.0714$, Wigley-hull 
SURGE HYDROSTATICS

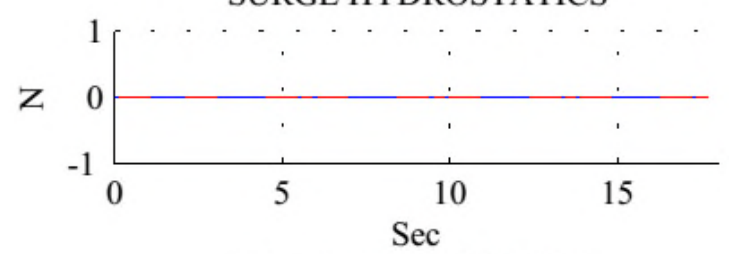

HEAVE HYDROSTATICS

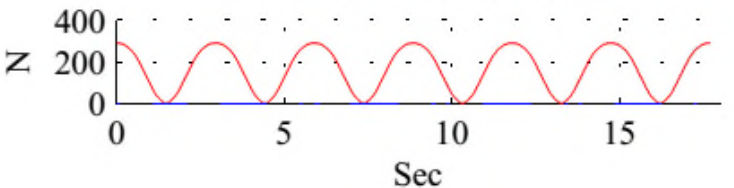

PITCH HYDROSTATICS

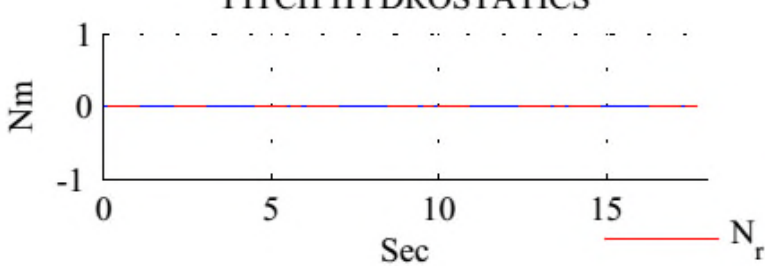

SWAY HYDROSTATICS
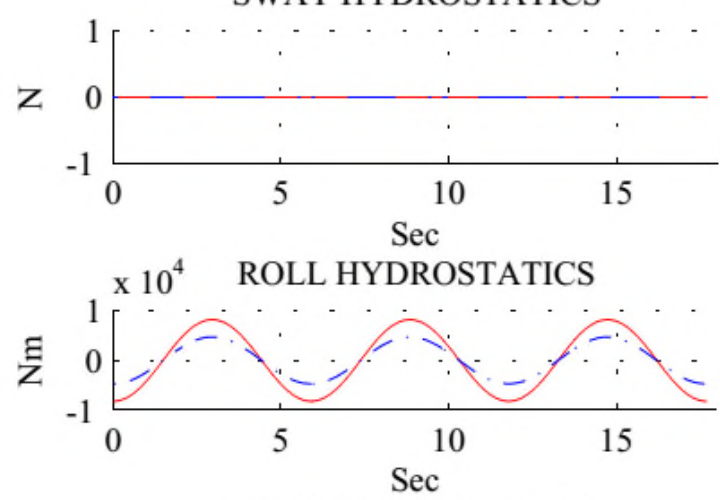

YAW HYDROSTATICS

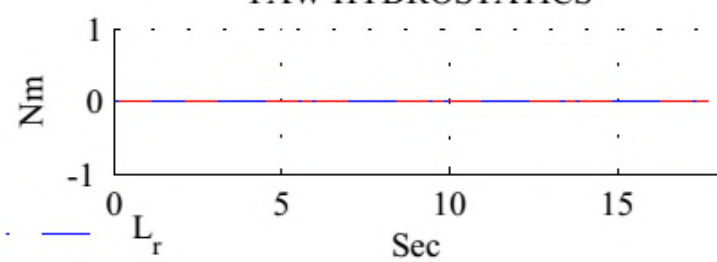

Figure 8: $0.05 \mathrm{rad}$ Forced roll hydrostatic, linear $L$ vs quasi-nonlinear $N, \omega_{e}=1.066 \mathrm{rad} / \mathrm{sec}, \mathrm{V}$ $=1 \mathrm{~m} / \mathrm{sec}, \mathrm{Fn}=0.0714$, Wigley-hull
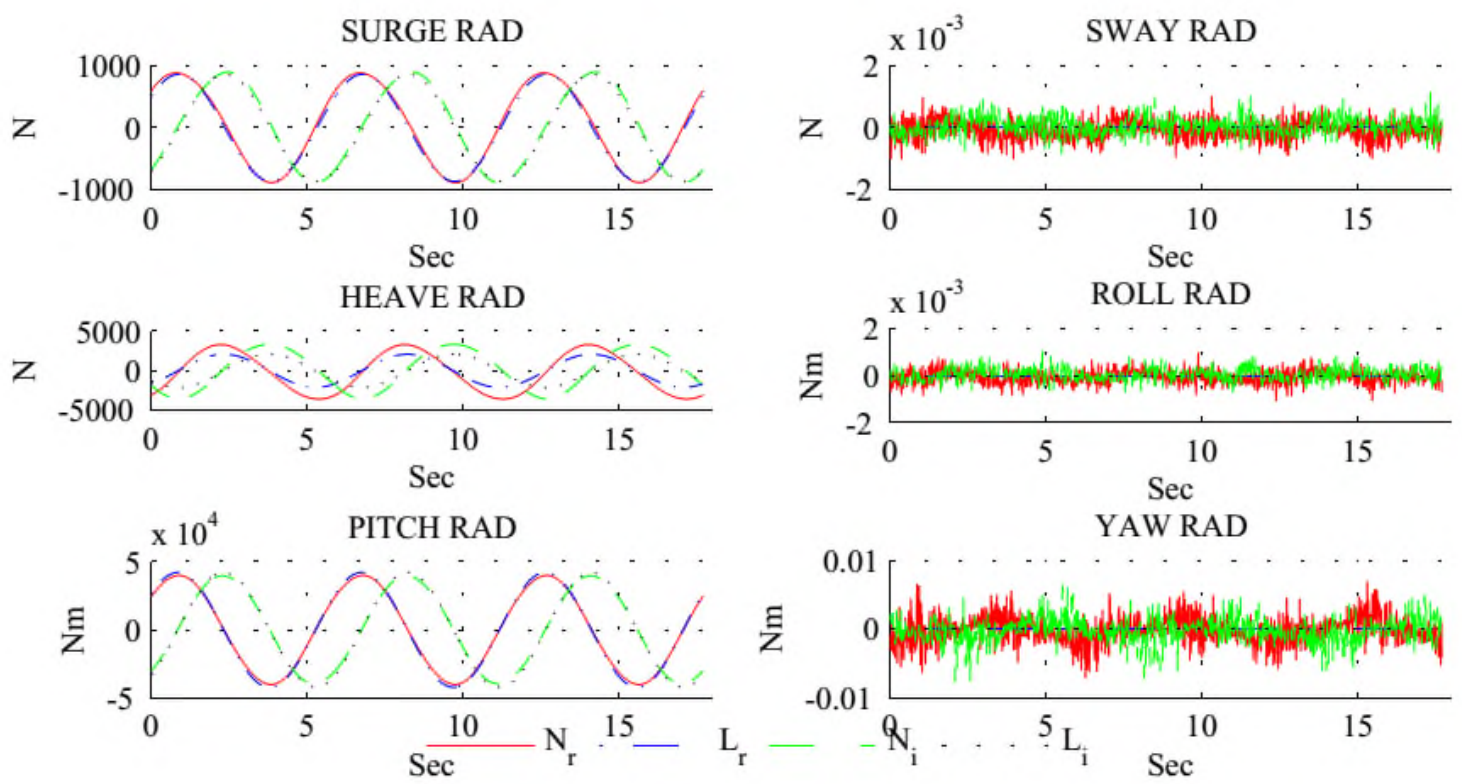

Figure 9: $0.05 \mathrm{rad}$ Forced pitch radiation, linear $L$ vs quasi-nonlinear $N, \omega_{e}=1.066 \mathrm{rad} / \mathrm{sec}, \mathrm{V}$ $=1 \mathrm{~m} / \mathrm{sec}, \mathrm{Fn}=0.0714$, Wigley-hull 
SURGE HYDROSTATICS

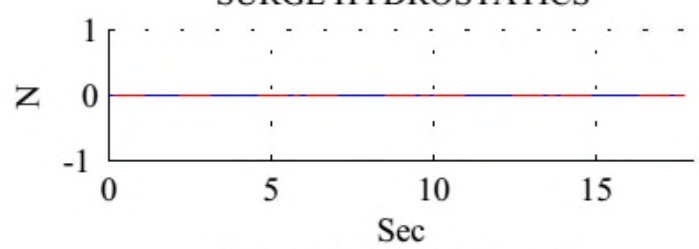

HEAVE HYDROSTATICS
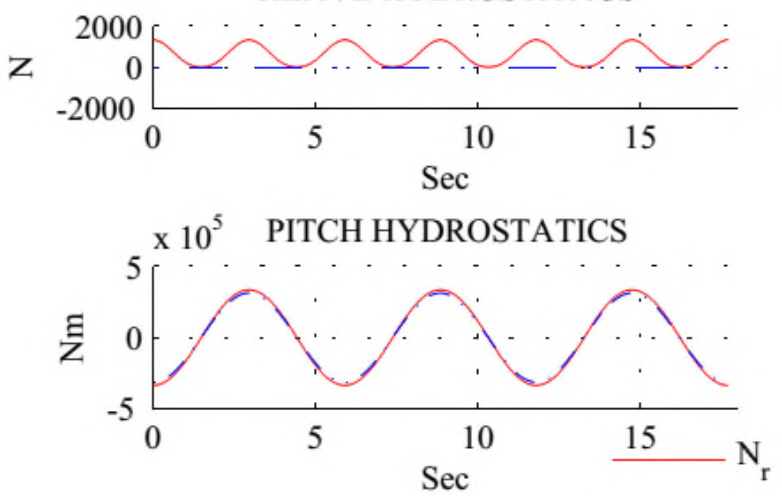

SWAY HYDROSTATICS

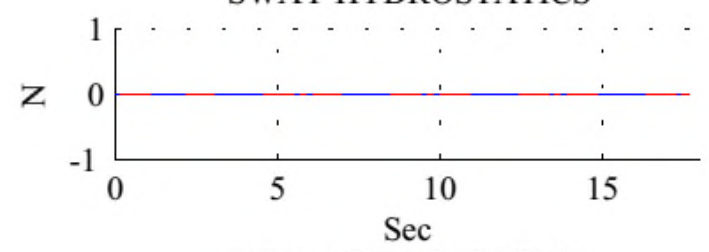

ROLL HYDROSTATICS

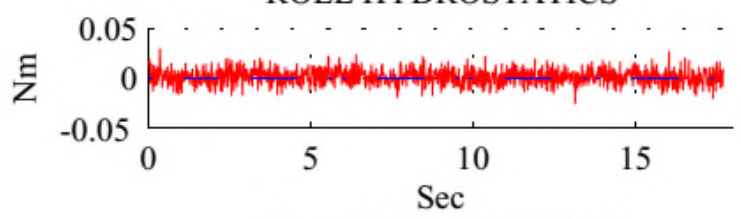

YAW HYDROSTATICS

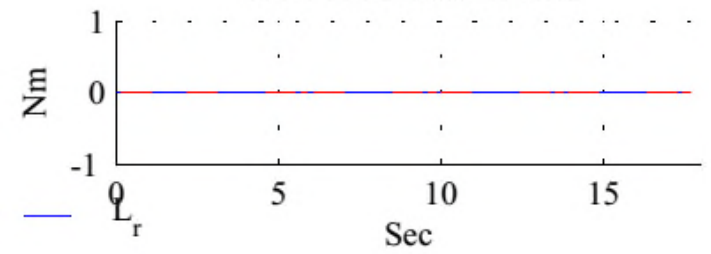

Figure 10: $0.05 \mathrm{rad}$ Forced pitch hydrostatic, linear $L$ vs quasi-nonlinear $N, \omega_{e}=1.066$ $\mathrm{rad} / \mathrm{sec}, \quad V=1 \mathrm{~m} / \mathrm{sec}, \mathrm{Fn}=0.0714$, Wigley-hull
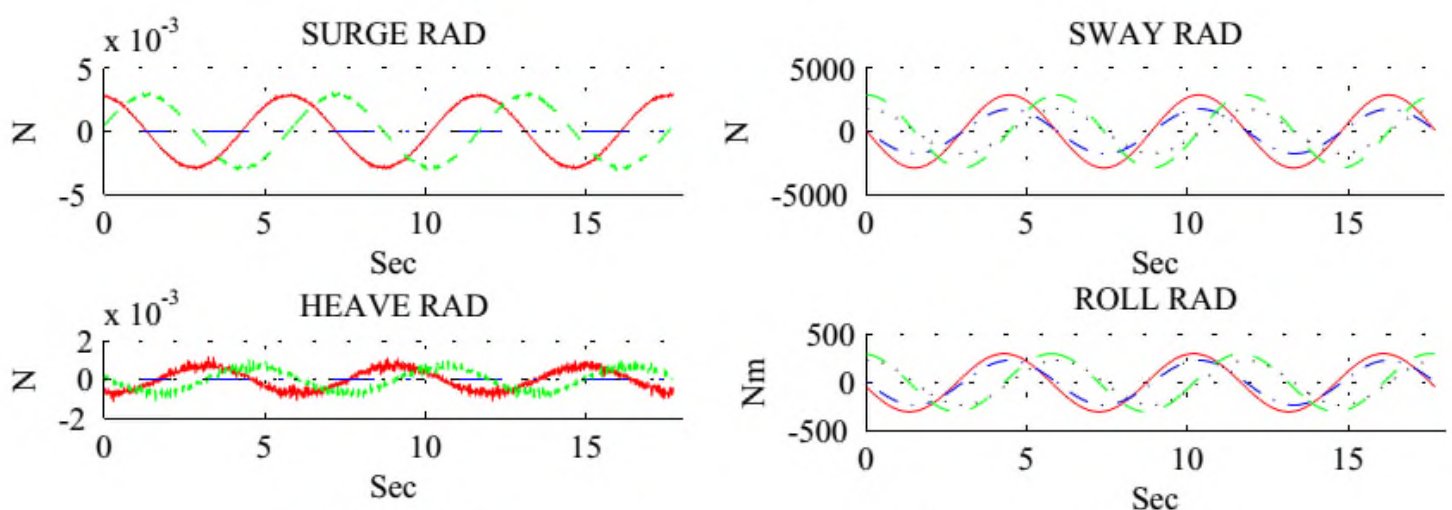

PITCH RAD
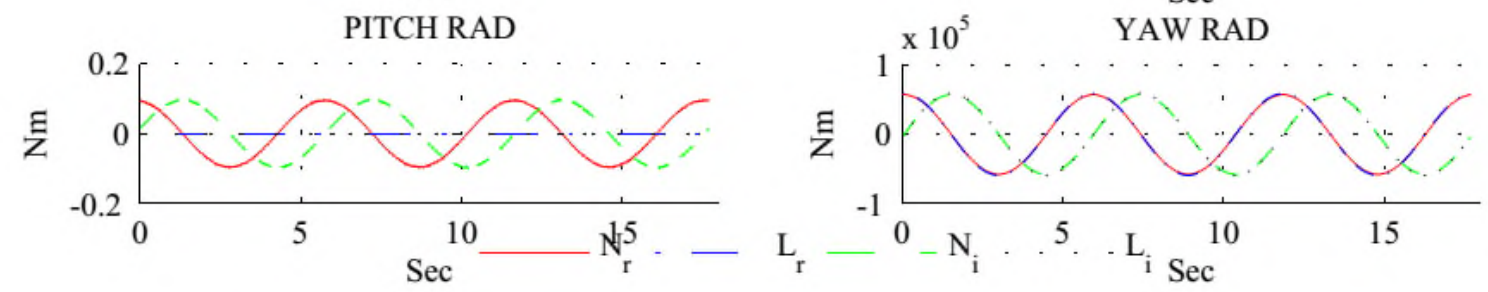

Figure 11: $0.05 \mathrm{rad}$ Forced yaw radiation, linear $L$ vs quasi-nonlinear $N, \omega_{e}=1.066 \mathrm{rad} / \mathrm{sec}$, $V=1 \mathrm{~m} / \mathrm{sec}, \mathrm{Fn}=0.0714$, Wigley-hull 
SURGE FK

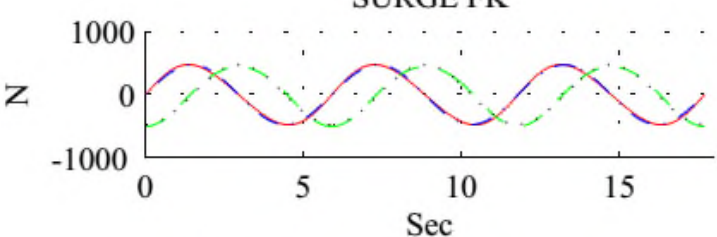

HEAVE FK

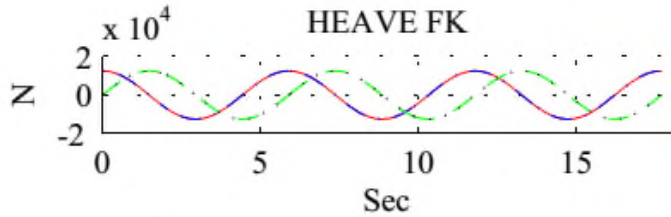

PITCH FK

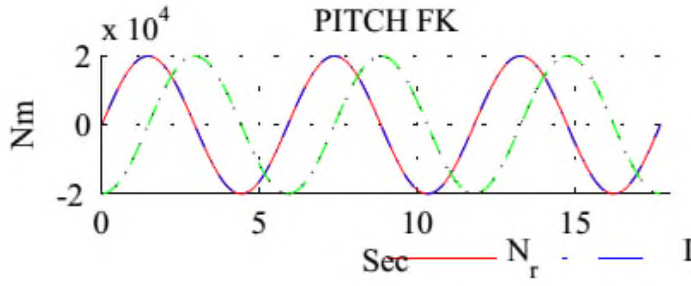

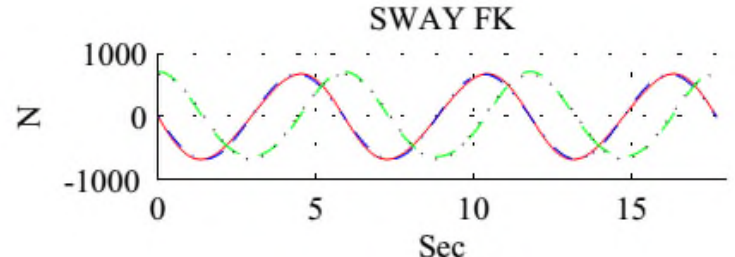

ROLL FK

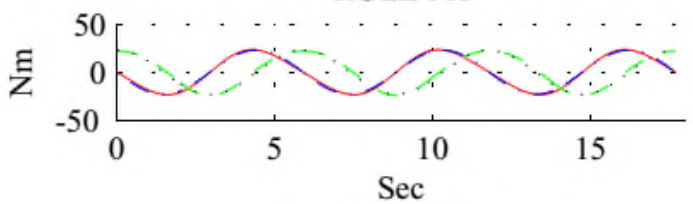

YAW FK

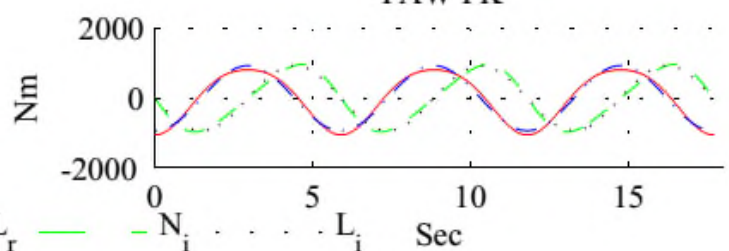

Figure 12: Froude-Kriloff forces, Linear $L$ vs quasi-nonlinear $N, \alpha=0.05 m, \beta=130^{\circ}, \omega=1$ $\mathrm{rad} / \mathrm{sec}, V=1 \mathrm{~m} / \mathrm{sec}, \mathrm{Fn}=0.0714$, Wigley-hull

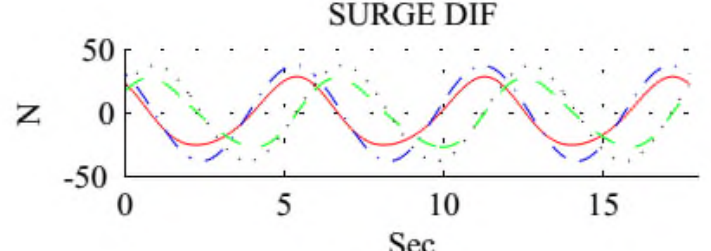

HEAVE DIF

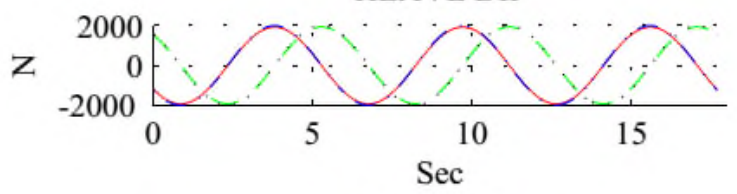

PITCH DIF

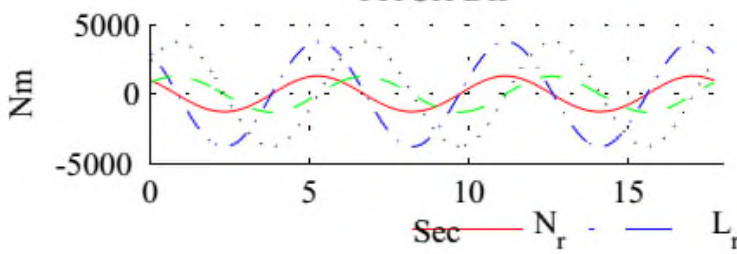

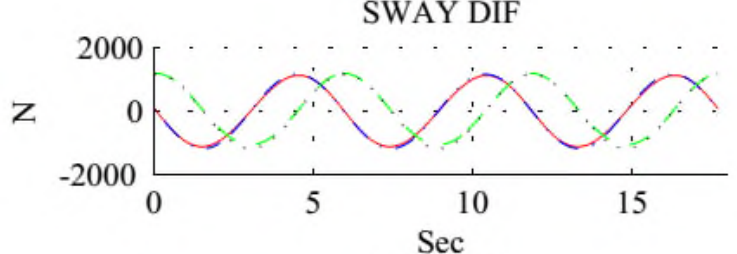

ROLL DIF

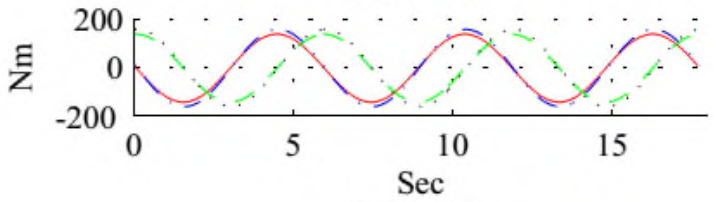

YAW DIF

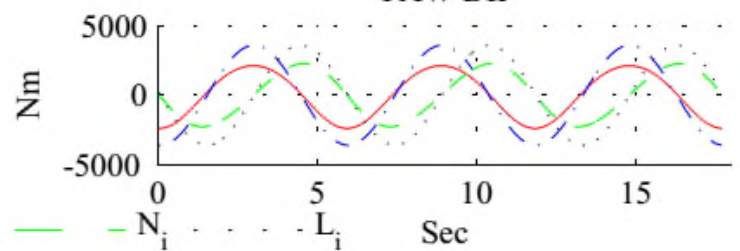

Figure 13: Diffraction forces, Linear $L$ vs quasi-nonlinear $N, \alpha=0.05 m, \beta=130^{\circ}, \omega=1$ $\mathrm{rad} / \mathrm{sec}, V=1 \mathrm{~m} / \mathrm{sec}, F n=0.0714$, Wigley-hull 

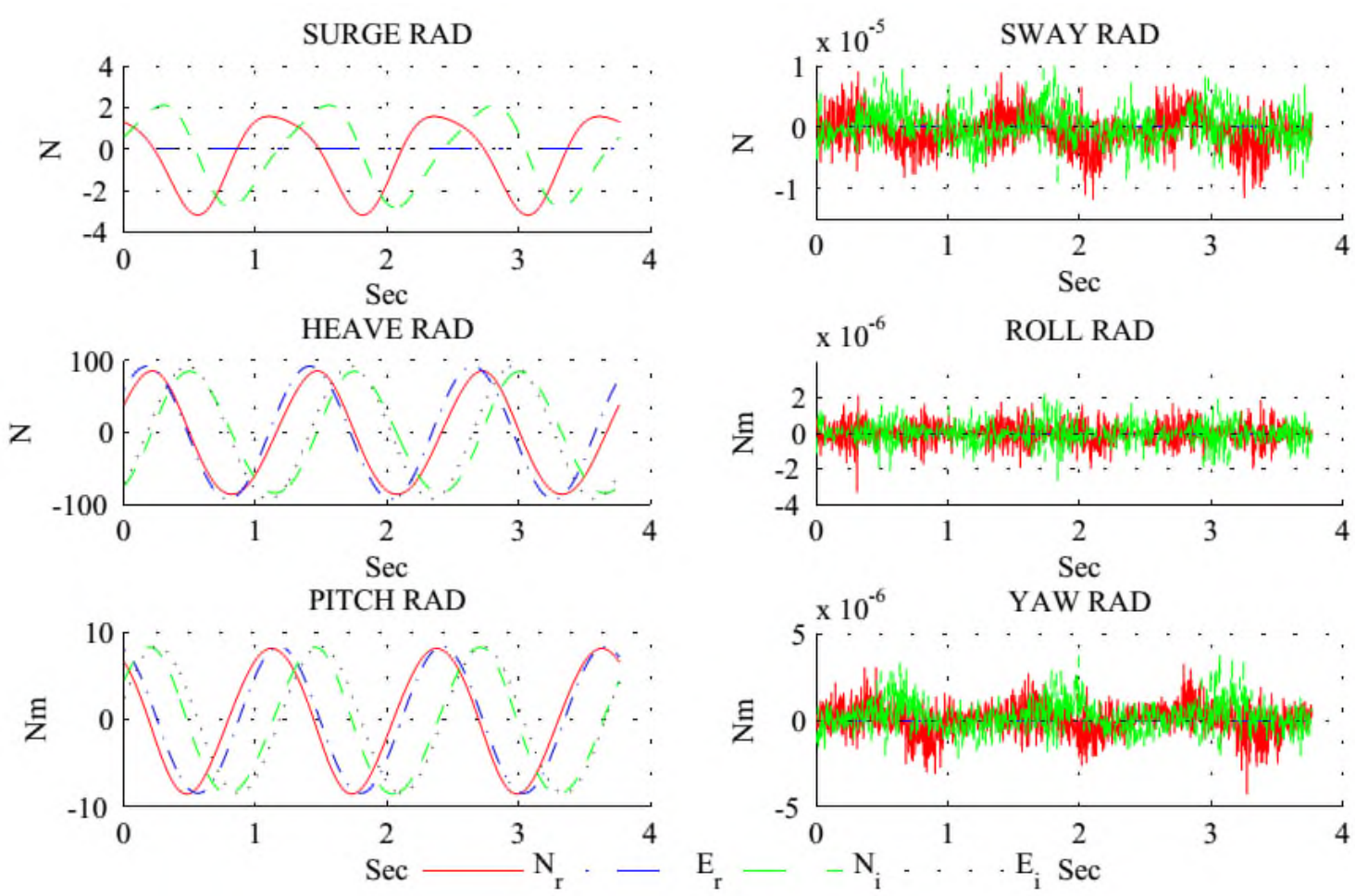

Figure 14: $0.05 \mathrm{~m}$ Forced heave radiation, experimental $E$ vs blended $N, \omega_{e}=5.0076 \mathrm{rad} / \mathrm{sec}$, $V=1.0848 \mathrm{~m} / \mathrm{sec}$, Wigley-hull-III
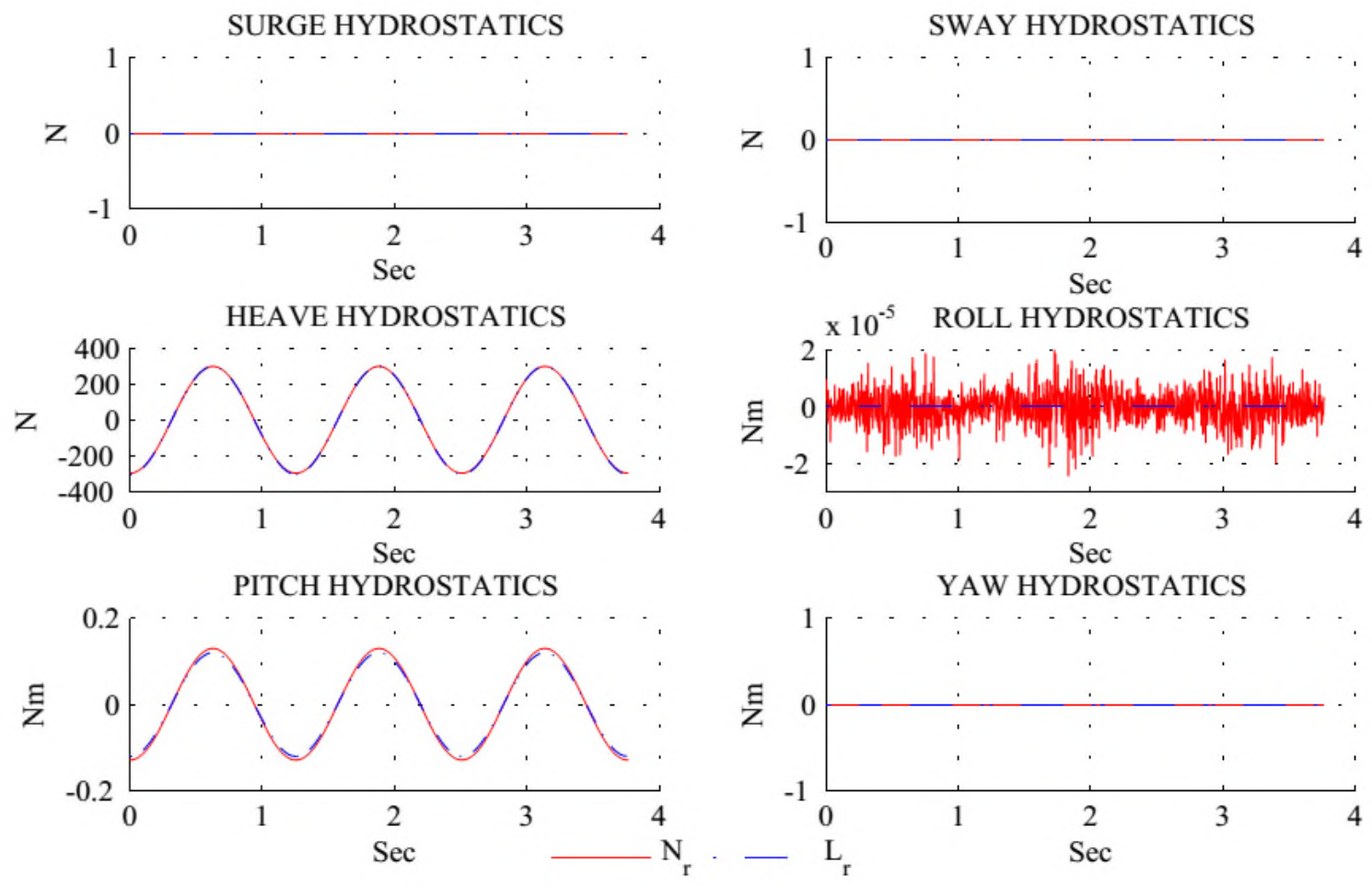

Figure 15: $0.05 \mathrm{~m}$ Forced heave hydrostatic, linear $L$ vs blended $N, \omega_{e}=5.0076 \mathrm{rad} / \mathrm{sec}, V=$ $1.0848 \mathrm{~m} / \mathrm{sec}$, Wigley-hull-III 

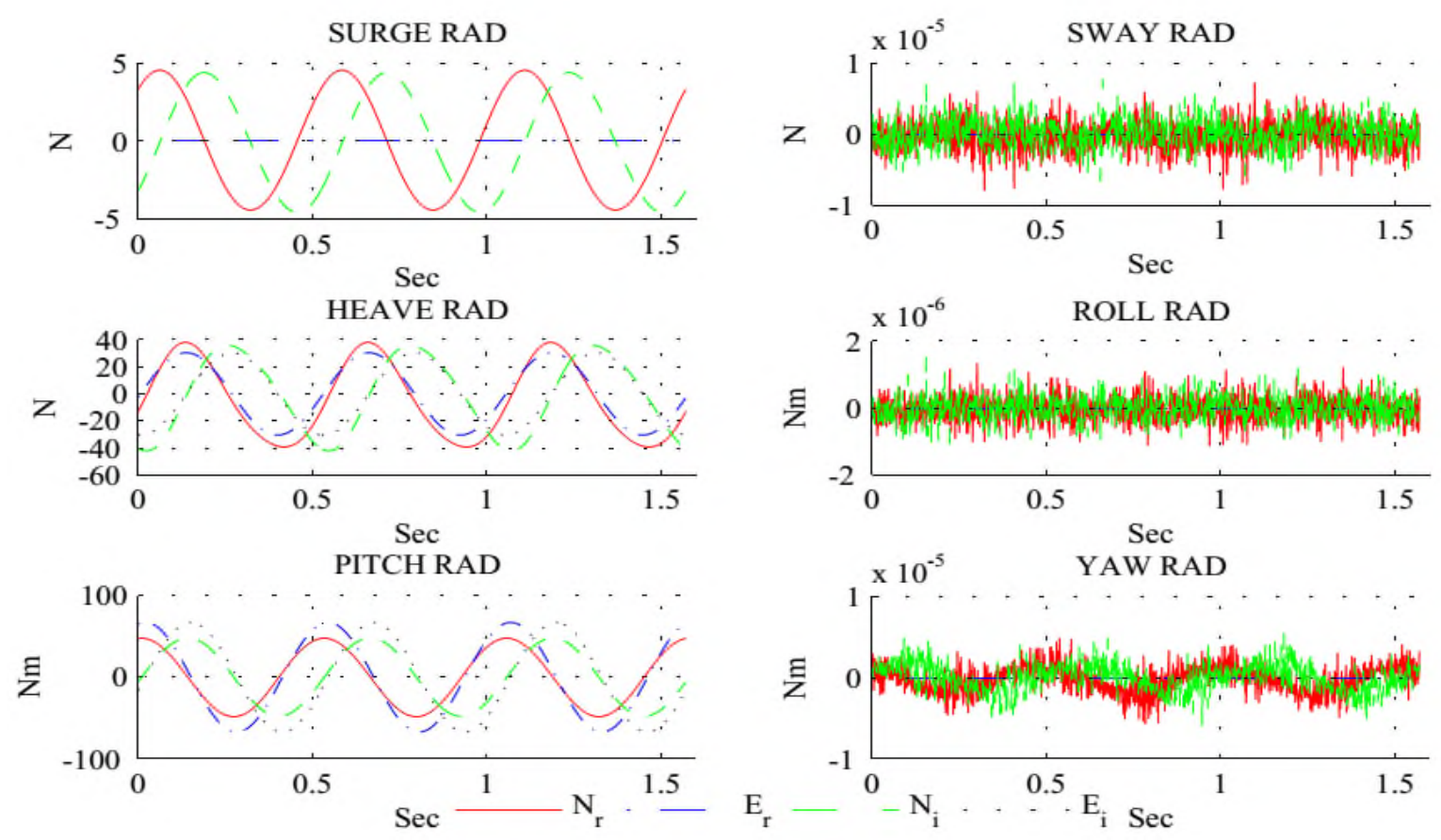

Figure 16: $0.0262 \mathrm{rad}$ Forced pitch radiation, experimental $E$ vs blended $N, \omega_{e}=12 \mathrm{rad} / \mathrm{sec}, \mathrm{V}$ $=2.1696 \mathrm{~m} / \mathrm{sec}$, Wigley-hull-III

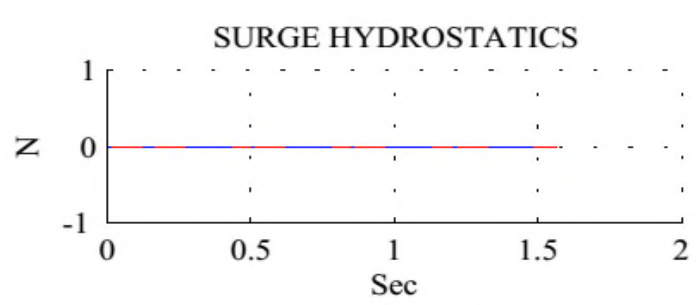

HEAVE HYDROSTATICS

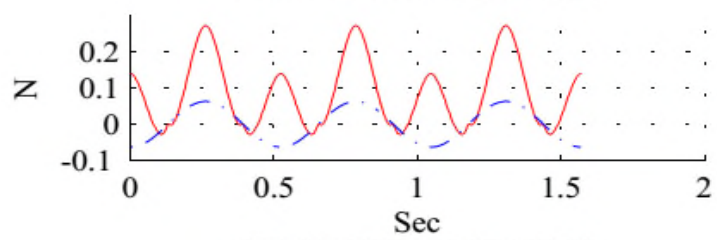

PITCH HYDROSTATICS
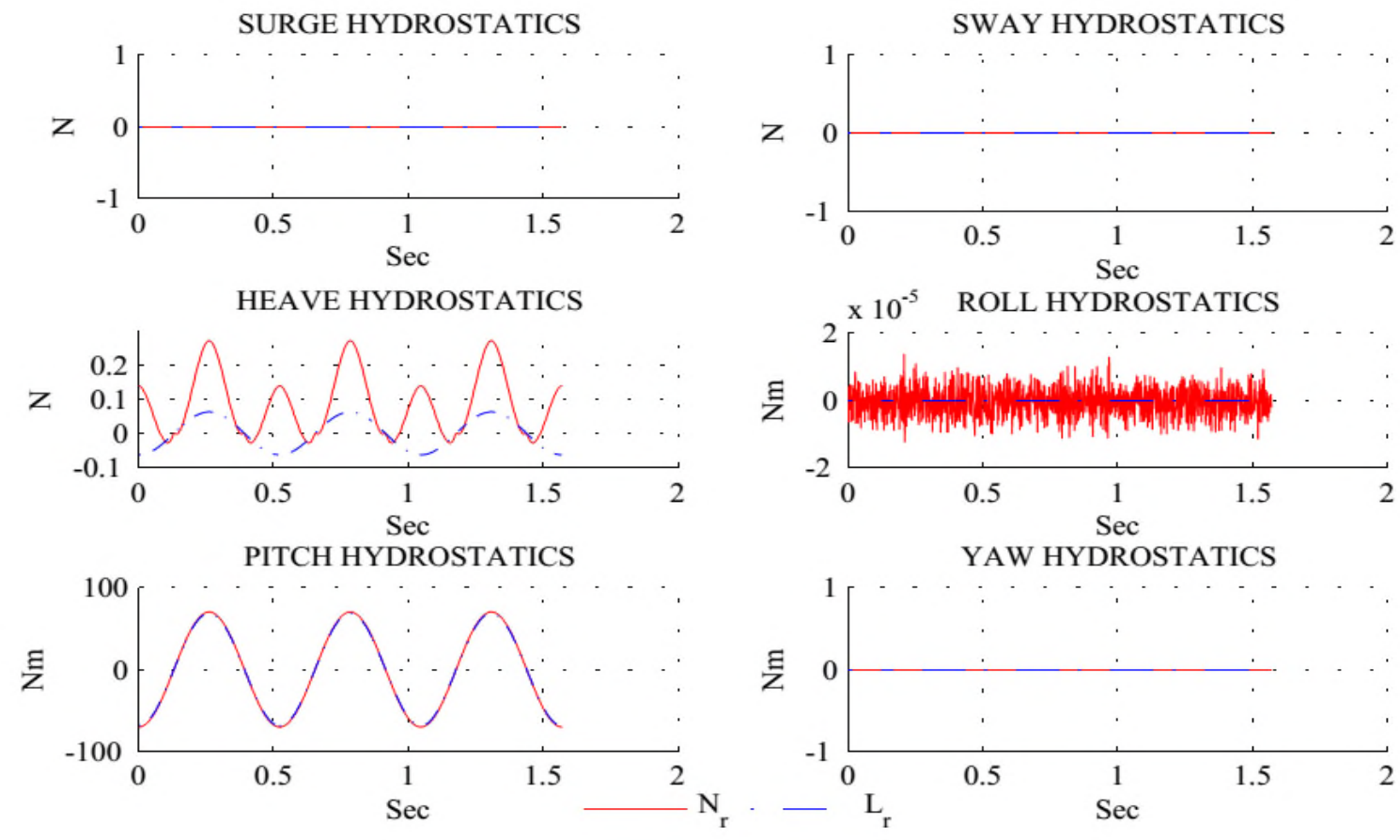

YAW HYDROSTATICS

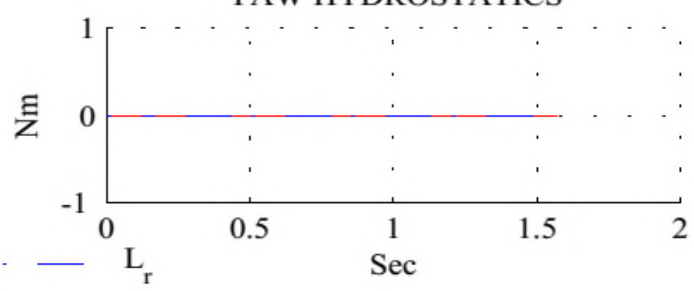

Figure 17: $0.0262 \mathrm{rad}$ Forced pitch hydrostatic, linear $L$ vs blended $N, \omega_{e}=12 \mathrm{rad} / \mathrm{sec}, \mathrm{V}=$ $2.1696 \mathrm{~m} / \mathrm{sec}$, Wigley-hull-III 

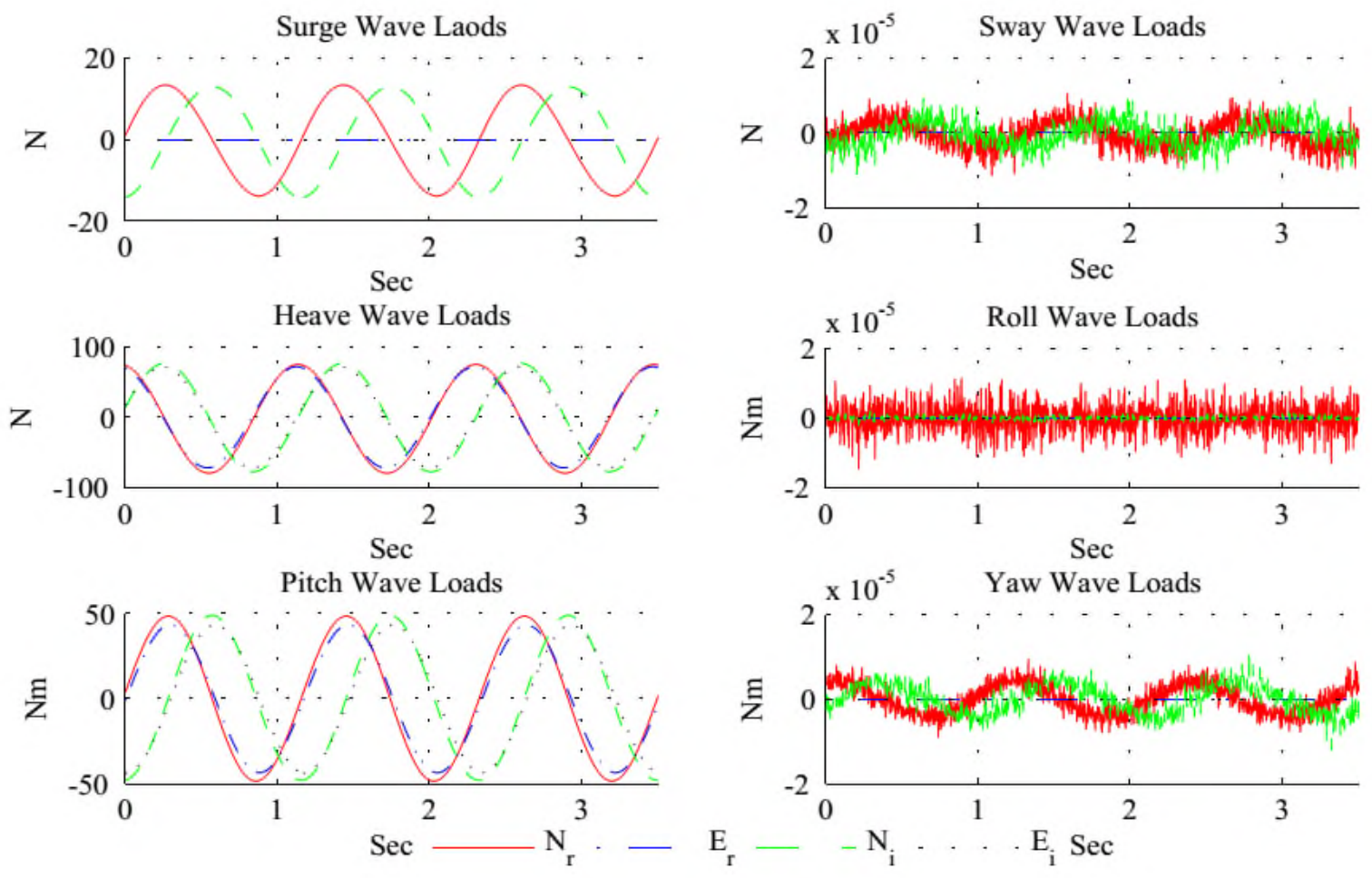

Figure 18: Wave loads for $\alpha=0.023 m$, experimental $E$ vs. blended $N, \beta=180^{\circ}, \omega=3.4259$ $\mathrm{rad} / \mathrm{sec}, \omega=5.4246 \mathrm{rad} / \mathrm{sec}, \mathrm{V}=1.6272 \mathrm{~m} / \mathrm{sec}$, Wigley-hull-III
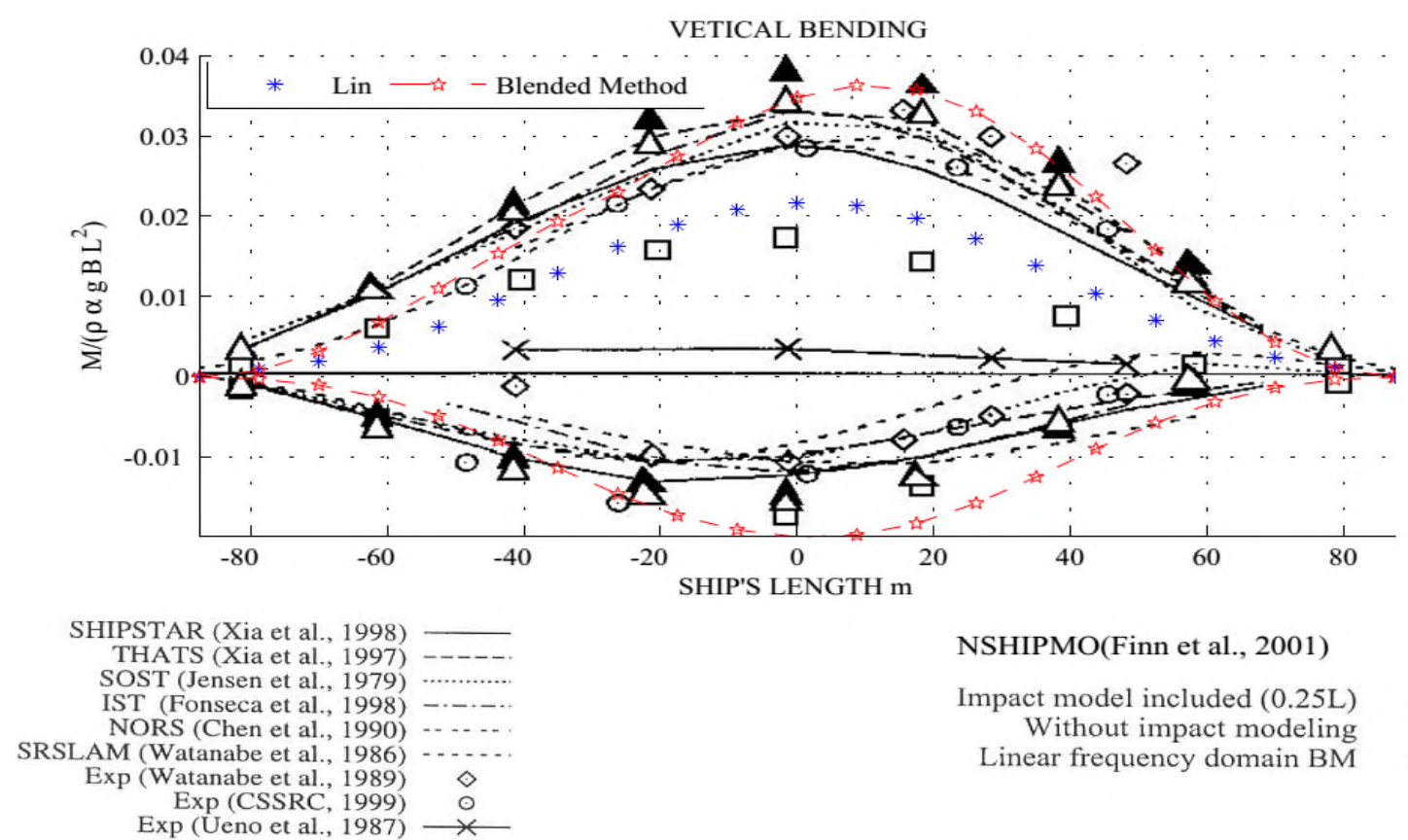

NSHIPMO(Finn et al., 2001)

Impact model included $(0.25 \mathrm{~L})$ $\begin{array}{cc}\text { Without impact modeling } & \mathbf{\Delta} \\ \text { Linear frequency domain BM } & \square\end{array}$

Figure 19: Nonlinear sagging $(M \geq 0)$ and hogging $(M \leq 0)$ bending moments of $S R 175$ container Ship for $\lambda=1.2 L, F_{n}=0.25, \alpha=L / 60$, old results extracted from [39] 
2020-02-01

\title{
Simulation of blended nonlinear
}

hydrodynamics forces using radial basis function in uniform moving frame

\author{
Khalid, Muhammed S.
}

Elsevier

Khalid MS, Nisar S, Khan SZ, et al., (2020) Simulation of blended nonlinear hydrodynamics forces using radial basis function in uniform moving frame. Ocean Engineering, Volume 198, February 2020, Article number 106994

https://doi.org/10.1016/j.oceaneng.2020.106994

Downloaded from Cranfield Library Services E-Repository 